\title{
DIMENSIONAL REDUCTION OF A MULTISCALE MODEL BASED ON LONG TIME ASYMPTOTICS
}

\author{
FRÉDÉRIQUE CLÉMENT*, FRÉDÉRIC COQUEL ${ }^{\dagger}$, MARIE POSTEL $^{\ddagger}$, AND KIM LONG \\ TRAN ${ }^{\ddagger}$
}

\begin{abstract}
We consider a class of kinetic models for which a moment equation has a natural interpretation. We show that, depending on their velocity field, some models lead to moment equations that enable one to compute monokinetic solutions economically. We detail the example of a multiscale structured cell population model, consisting of a system of $2 \mathrm{D}$ transport equations. The reduced model, a system of $1 \mathrm{D}$ transport equations, is obtained by computing the moments of the $2 \mathrm{D}$ model with respect to one variable. The $1 \mathrm{D}$ solution is defined from the solution of the $2 \mathrm{D}$ model starting from an initial condition that is a Dirac mass in the direction removed by reduction. For arbitrary initial conditions, we compare 1D and 2D model solutions in asymptotically large time. Finite volume numerical approximations of the $1 \mathrm{D}$ reduced model can be used to compute the moments of the $2 \mathrm{D}$ solution with proper accuracy. The numerical robustness is studied in the scalar case, and a full scale vector case is presented.
\end{abstract}

Key words. model reduction, kinetic models, cell structured population, finite volume method, asymptotic behavior

AMS subject classifications. 35Q92, 35B40, 65N08

1. Introduction. In this article we derive the dimensional reduction of a multiscale structured cell population model [16], consisting of a system of transport equations, whose solution describes the density of cells as a function of time, age within the cell cycle and maturity. We address the reduction issue within the scope of kinetic models, more precisely of systems of equations whose unknowns depend not only on time and space (time and cell age in our case), but also on "kinetic" variables: instantaneous speed, local energy level, or, in our setup, the cell maturity variable. Usually observable states are moments of the solution with respect to kinetic variables and are therefore function of time and space, indeed of time only in many cases.

While the kinetic formulation of gas dynamics is studied theoretically in [28], many applications in various fields are described in the literature, along with specific numerical methods, modeling for instance fluid dynamics [25], strain and stress in mechanical or biomechanical models [5], production models [21], crowd [13, 33] models, predator-prey [14] and other biological systems [29].

In most real life applications, measurements are scarce and correspond to first order moments. A system of PDEs that would directly govern the dynamics of these selected moments is of course very attractive. The main interest of such a reduced model is the CPU saving which comes from the reduction of dimension, even though the number of equations may increase. Numerical simulation of the moment model will be much faster than the initial model and therefore more adapted for control purposes or parameter identification based on moment estimations.

Such a reduced model is however difficult to obtain in the general case. The whole infinite hierarchy of moments naturally arises when the initial PDEs are multiplied by

*INRIA Paris, Mycenae, 2 rue Simone Iff, F-75589 Paris Cedex 12, France

${ }^{\dagger}$ CMAP UMR 7641 École Polytechnique CNRS, Route de Saclay, F-91128 Palaiseau Cedex, France

¥Sorbonne Universités, UPMC Univ Paris 06, UMR 7598, Laboratoire Jacques-Louis Lions, F75005, Paris, France

$\S$ (Marie.Postel@upmc.fr) 
the microscopic variable to an arbitrary power before being integrated and can lead to complicated models [32], requiring an elaborate theory to be stabilized [27, 22].

A priori knowledge is required to relate the higher order moments to the interesting ones and close this infinite system. Even though, the reduced models themselves yield interesting existence problems $[24,23]$ when the solutions are functions. Other models of a collision nature, developped in the context of "sticky particles" [9] or pressureless gas dynamics [6] yield moment models whose solutions are measures for regular initial conditions.

Let us also underline the work [19] devoted to a numerical study of the semiclassical approximation of the motion of electrons in short-scale periodic potentials. The authors put forward that the usual approach results in a moment system which is weakly hyperbolic and thus also exhibits measure valued solutions. An elegant alternative strategy is presented, which relies on multiphase WKB expansions combined with homogenization procedures. The authors obtain a moment system with multi valued solutions whose relevant definition and approximation relies on the K-branch solutions first introduced in [8].

Here we deal with kinetic equations (including birth and death phenomena) whose dynamics are ruled by a velocity field which is nonlinear in the kinetic variables. We tackle the closure difficulty thanks to an action principle of return to the equilibrium. The knowledge of a distribution function for the solution in asymptotically long time enables us to estimate higher order moments at intermediate times. For a specific smooth velocity field, this approximation yields a reduced model whose solution is defined for all times and can be used to design a measure solution starting from a measure initial condition [26].

The problem that we address here encompasses discontinuous velocity fields, associated with well known ill posedness issues [12,7], and the consequent numerical difficulties [18]. On the one hand, existence and uniqueness of the solution in our case is proven for bounded initial conditions, and hence as well the existence of zeroth and first order moments [30]. On the other hand, the same asymptotic behavior as in the smooth velocity case [26], tending towards a monokinetic distribution, is observed in numerical simulations $[2,4]$. Nevertheless, we will show that if we relax the hypotheses made in [26] on the velocity field, the reduced model can have only measure solutions in some cases, and is therefore of little use for practical and numerical purposes, while in other cases the monokinetic behavior [26] is preserved. The cell population model of interest $[16,11]$ falls into the first case. However, we will show that if we integrate its zeroth and first order moments over all space variables, the resulting time functions are identical (for linear velocity fields) or numerically close (for quadratic velocity fields) to the integrated moments obtained from an equivalent model for which a "useful" reduced model can be derived using the monokinetic behavior.

The paper is organized as follows. In section 2 we illustrate the main tools on a 1D toy model: long time asymptotic behavior of the transport equation (Theorem 2.1 ), moment-based reduction method (Theorem 2.2). We then recall the $2 \mathrm{D}$ cell population kinetics model and apply the moment method to derive a system of $1 \mathrm{D}$ PDEs. We show that, in our case, this reduced system of 1D equations is ill-posed due to the discontinuity of the $x$-velocity in the orthogonal direction $y$. To tackle this difficulty we introduce a modified $2 \mathrm{D}$ set-up and show that it is equivalent -to some extent- to the original one in term of zeroth and first moments of the solution. Theorem 2.3 establishes the theoretical result in a simplified situation, while numerical 
simulations illustrate its robustness when extended to the actual model. In section 3 , we describe and study mathematically the reduction of the model from $2 \mathrm{D}$ to $1 \mathrm{D}$ for this new set-up. Theorem 3.1 and Lemma 3.2 exhibit a solution of the reduced model built from the monokinetic solution of the $2 \mathrm{D}$ problem. We then prove in Theorem 3.3 that the cell density converges for long time towards a measure-valued solution in maturity, by bracketing the support of the 2D solution with particular solutions of the reduced model. In section 4, we introduce the numerical scheme designed to solve the reduced model and study its robustness and accuracy. In section 5, we compare the reduced model solution with the full $2 \mathrm{D}$ one on the macroscopic scale, first in the scalar case of a single cell population and then in the case of multiple and interacting populations.

\section{Reduction based on long time asymptotic behavior.}

2.1. 1D toy model. In order to present more clearly the model reduction technique, we first perform it on a simple one dimensional PDE

$$
\left\{\begin{array}{l}
\partial_{t} \phi(t, y)+\partial_{y}(h(t, y) \phi(t, y))=0 \\
\phi(0, y)=\phi_{0}(y)
\end{array}\right.
$$

We intend to exhibit an ordinary differential equation whose solution is the first order moment in $y$ of the solution of (2.1). We assume that the initial condition $\phi_{0}$ is a positive function -or Dirac measure- with compact support

$$
\left.\operatorname{Supp}\left(\phi_{0}\right) \subset\right] y_{\min }^{0}, y_{\max }^{0}[\text {, }
$$

and that the velocity $h(t, y)$ is $C^{1}\left(\left[0,+\infty[\times \mathbb{R})\right.\right.$ and satisfies ${ }^{1}$

$$
\begin{gathered}
h(0, y)>0, \forall y \in] y_{\min }^{0}, y_{\max }^{0}[, \\
\partial_{y} h(t, y) \leq-\eta<0, \forall y \geq y_{\min }^{0} .
\end{gathered}
$$

Defining the zeroth and first order moments

$$
M_{0}(t)=\int_{\mathbb{R}} \phi(t, y) \mathrm{d} y, \quad M_{1}(t)=\int_{\mathbb{R}} y \phi(t, y) \mathrm{d} y
$$

we obtain that $M_{0}(t)=M_{0}$ remains constant by integrating (2.1) on $\mathbb{R}$. Then multiplying (2.1) by $y$ before integrating leads to

$$
\frac{d}{d t} M_{1}(t)=\int_{\mathbb{R}} h(t, y) \phi(t, y) \mathrm{d} y
$$

At this point we need an additional hypothesis to close equation (2.6). We will rely on the asymptotic behavior of the solution of (2.1) for $t \rightarrow+\infty$, which can be expressed in terms of the characteristic curves and is described by the following theorem

THEOREM 2.1. Let $\phi(t, y)$ satisfy (2.1,2.2) and let $\zeta_{1}(t), \zeta_{2}(t)$ be the solutions of

$$
\left\{\begin{array}{l}
\frac{d}{d t} \zeta(t)=h(t, \zeta(t)), \\
\zeta(0)=\zeta_{0},
\end{array}\right.
$$

\footnotetext{
${ }^{1}$ For instance $h(t, y)=\eta\left(y_{\max }^{0}-y\right)$ satisfies $(2.3-2.4)$.
} 


$$
\begin{aligned}
& \text { for } \zeta_{1}(0)=y_{\min }^{0} \text { and } \zeta_{2}(0)=y_{\max }^{0} \text {. Then } \\
& \operatorname{Supp}(\phi(t, .)) \subset\left[\zeta_{1}(t), \zeta_{2}(t)\right] \\
& \left|\zeta_{2}(t)-\zeta_{1}(t)\right| \leq e^{-\eta t}\left|y_{\max }^{0}-y_{\min }^{0}\right| .
\end{aligned}
$$

The proof of this theorem is postponed to Appendix A.

The fact that the width of the support of the solution goes to zero when $t \rightarrow+\infty$ motivates the study of a specific solution for (2.1), which would satisfy this property also for finite time. As mentioned in the introduction, the monokinetic hypothesis for the equilibrium solution is a standard tool in other fields as in compressible turbulence, and used for instance in [10] to describe pressure-less gas dynamics. Using this tool here yields the following result

TheOREM 2.2. Let $M_{0}$ be a positive constant and $\phi^{a}(t, y)=M_{0} \delta(y-\zeta(t))$ the measure built from $\zeta(t)$, the solution of (2.7) for any initial value $\zeta_{0}$. Then $\phi^{a}(t, y)$ is a weak solution of (2.1) with initial condition $\phi_{0}(y)=M_{0} \delta\left(y-\zeta_{0}\right)$.

The proof of this result (also postponed to Appendix A) is straightforward. Furthermore the first moment is $M_{1}(t)=\int_{\mathbb{R}} y \phi^{a}(t, y) \mathrm{d} y=M_{0} \zeta(t)$ therefore (2.6) boils down to (2.7), which is the sought-after ODE.

Remarks: (i) In the 1D model derived from our biological application, the time dependency of the velocity arises from a non local feedback control : $h(t, y)=$ $\tilde{h}\left(M_{1}(t), y\right)$. The theoretical study of $(2.1)$ is then more involved. A similar problem for $h(t, y)=\tilde{h}\left(M_{0}(t), y\right)$ is studied in [31].

(ii) The reduction of the toy model highlights the dependency of the approximation on the width of the initial condition support $\left[y_{\min }^{0}, y_{\max }^{0}\right]$, and on $\eta$, the decreasing rate of the velocity. We will propose in Paragraph 5.3 a refined method in the case when the initial condition has an arbitrarily wide support. The decreasing velocity hypothesis (2.4), is necessary to obtain the weak convergence of the solution $\phi(t, y)$ towards the measure $\phi^{a}(t, y)$ when $t \rightarrow \infty$. Yet it can be relaxed to $\eta=0$ and we will still have the bounds (2.8) on the support of $\phi(t,$.$) .$

2.2. Multiscale 2D model. We now focus on multiscale models describing structured cell population dynamics, whose mathematical properties are quite generic in the framework of transport problems. On the microscopic level, cells are described by their density expressed as a function of time and two functional variables: $x$, the age within the cell cycle and $y$, the cell maturity. A vector of cell densities describing interacting populations is the solution of a system of coupled transport equations. The equations are weakly coupled through control terms which depend on some moments of the solution. The age variable, $x$, and its associated velocity, $g_{f}(t, x, y)$, accounts for time variations or feed back induced variations of the cell cycle duration not only with respect to time but also from one population to the other. The cell maturity variable, $y$, discriminates different cell states (or fates): proliferation, differentiation, cell death. Other multiscale models describe biological populations with respect to age and spatial variables or other functional variables such as size for instance [15]. An important and original feature of the model is that the domain of definition is subdivided into phases corresponding to these cell states. The transport velocities and source terms are time and space dependent piecewise continuous functions and may present discontinuities on the inner boundaries. Moreover, the cell division (mitosis ${ }^{2}$ ) at the end of each cell cycle is modeled by a doubling of the flux in the age direction on the outer boundary of the cell cycle $\left(y<y_{s}\right)$. In the differentiated domain $\left(y>y_{s}\right)$

\footnotetext{
${ }^{2}$ Mitosis is the last step of the cell cycle where two daughter cells are born from a mother cell.
} 
the cell density is periodic in $x$. The consequences of the velocity discontinuities on the well-posedness of the model have been tackled in [30], where the existence and uniqueness of the solution is obtained, while a specific numerical treatment has been designed in [2] and [4]. Several strategies have already been explored to reduce the computational costs (parallel computing[1], adaptive mesh refinement [4]).

We denote by $N_{f}$ the number of interacting cell populations. For $f=1, \ldots, N_{f}$, the time evolution of the cell density $\Phi_{f}(t, x, y)$ is given as the solution in $L^{\infty}\left(\mathbb{R}^{+} \times \Omega ; \mathbb{R}^{N_{f}}\right)$ to the following first order PDE equations:

$$
\begin{aligned}
\frac{\partial}{\partial t} \Phi_{f}(t, x, y)+\frac{\partial}{\partial x}\left(g_{f}(t, x, y) \Phi_{f}(t, x, y)\right) & +\frac{\partial}{\partial y}\left(h_{f}(t, x, y) \Phi_{f}(t, x, y)\right) \\
& =-\Lambda(t, x, y) \Phi_{f}(t, x, y) .
\end{aligned}
$$

The computing space domain $(x, y) \in \Omega=\sum_{i} \Omega_{i}$ is the union of a finite number of open sets. In the original model [16], that we present in this paragraph, $\Omega=$ $G 1 \cup S M \cup D^{3}$, with

$$
\left\{\begin{array}{l}
G 1=\left\{(x, y), 0<x<x_{1}, 0<y<y_{s}\right\}, \\
S M=\left\{(x, y), x_{1}<x<x_{2}, 0<y<y_{s}\right\}, \\
D=\left\{(x, y), y_{s}<y\right\}
\end{array}\right.
$$

where each subset corresponds to a different cell state as illustrated on the left panel of Figure 2.1. The variations of the velocities and source term, which are $C^{1}$ in time

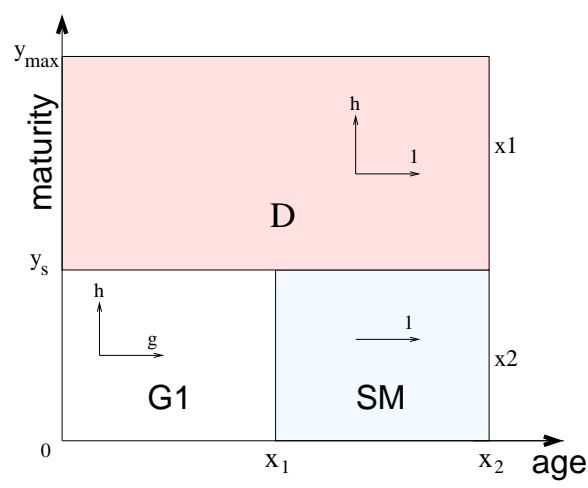

a) The original set-up (2.11)

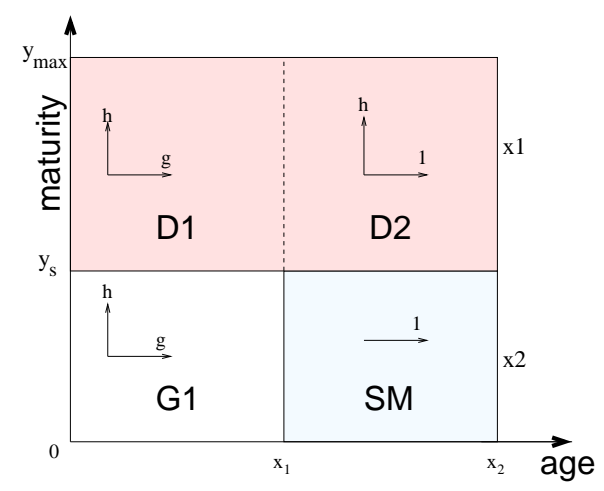

b) The modified set-up (2.30)

FIG. 2.1. Computing domain $\Omega$. The left panel describes the structure of the domain for the original multiscale model (2.10-2.11) with the corresponding boundary and transmission conditions (2.12-2.16). The right panel illustrates the changes for the modified set-up described in equations (2.10)-(2.30), (2.12,2.13,2.16,2.31,2.32). Notice the separation of the D phase into two D1 and D2 subphases and the $g$ velocity in phase $D 1$. The upper bound $y_{\max }$ is chosen such that no cell can reach the top of the domain within $t \leq t_{\max }$, the duration of the simulation.

and on each subset of $\Omega$ in space, are depicted in Figure 2.1. We assume that the vertical velocity $h_{f}$ and source term $\Lambda$ are null in the subdomain $S M$ and functions of $t$ and $y$ elsewhere. The vertical velocity $h_{f}$ is positive for all times in $G 1$ and has at

\footnotetext{
${ }^{3} \mathrm{G} 1$ and D correspond to different phases of the cell division cycle. This cycle is divided into G1,S, G2 and M phases. In the model we distinguish the G1 phase (in which the cell is sensistive to extracellular signals from the remaining phases that are gathered under the denomination SM. D stands for Differentiated and is used for the cells having exited the cell cycle. .
} 
least one zero in $D$. The horizontal speed $g_{f}$ is constant equal to 1 in $S M$ and $D$ and a function of $t$ only in $G 1$. Therefore $h_{f}$ is discontinuous on the boundaries between $S M$ and $G 1 \cup D$ and $g_{f}$ is discontinuous on the boundaries between $G 1$ and $S M \cup D$. The system (2.10) is completed with outer and inner boundary conditions wherever $\bar{\Omega}_{i} \cap \bar{\Omega}_{j} \neq \emptyset$ : We suppose that in the lower (proliferative) part of the domain, the flux on the $x$-axis is continuous on the inner boundary between $G 1$ and $S M$ phases

$$
\Phi_{f}\left(t, x_{1}^{+}, y\right)=g_{f}\left(t, x_{1}^{-}, y\right) \Phi_{f}\left(t, x_{1}^{-}, y\right), \quad 0<y<y_{s},
$$

and satisfies a doubling boundary condition at the end of the cell cycle, modeling mitosis

$$
g_{f}\left(t, 0^{+}, y\right) \Phi_{f}\left(t, 0^{+}, y\right)=2 \Phi_{f}\left(t, x_{2}^{-}, y\right), \quad 0<y<y_{s} .
$$

In the upper (differentiated) part of the domain, the $x$ directional flux satisfies a periodic condition

$$
\Phi_{f}\left(t, 0^{+}, y\right)=\Phi_{f}\left(t, x_{2}^{-}, y\right), \quad y>y_{s} .
$$

Thanks to the continuity of $h_{f}(t, x, y)$ with respect to $y$, the boundary condition between phases $G 1$ and $D$ (through the threshold maturity $y_{s}$ ) is given by

$$
\left.\Phi_{f}\left(t, x, y_{s}^{+}\right)=\Phi_{f}\left(t, x, y_{s}^{-}\right), \quad x \in\right] 0, x_{1}[.
$$

Finally, we apply a homogeneous Dirichlet condition to the north of the interface $S M-D$

$$
\left.\Phi_{f}\left(t, x, y_{s}^{+}\right)=0, \quad x \in\right] x_{1}, x_{2}[.
$$

The initial condition is given by the density in age $x$ and maturity $y$ of the cells at initial time

$$
\Phi_{f}(0, x, y)=\Phi_{0}(x, y), \text { with } \operatorname{Supp}\left(\Phi_{0}\right)=\left[0, x_{2}\right] \times\left[y_{0}^{\min }, y_{0}^{\max }\right] \subset G 1 \cup S M .
$$

This, along with the positivity of $h_{f}$ at all times in $G 1 \cup S M$, ensures that

$$
\left.\Phi_{f}(t, x, y)=0, \quad t \geq 0, \quad x \in\right] 0, x_{2}[, \quad y \leq 0,
$$

which enables us to consider only positive $y$ in the sequel. The existence and uniqueness of the solution of (2.10) along with initial condition (2.17) and limit conditions $(2.12,2.13,2.14,2.15,2.16,2.18)$ have been obtained in [30] for the particular choice of velocities and source term that we will consider for the numerical applications in the sequel. In our biological framework, the time dependency of these coefficients also establishes a weak coupling between the $N_{f}$ equations through the first moment in $y$ of their solutions. The proof in [30] relies on a fixed point argument to match the solution established for general velocity and source terms with the particular closed loop control solution.

The remaining of the paper endeavours to generalize the 1D reduction method presented in the previous paragraph to the $2 \mathrm{D}$ problem (2.10). The space dependency of the velocities $g_{f}$ and $h_{f}$ prevents us from simplifying the problem to (2.1) and the moment equations remain one dimensional PDEs

The originality of our work lies in its addressing the well-posedness issue of the reduced model in the case of $2 \mathrm{D}$ transport equations with discontinuous coefficients. 
This issue arises whenever the velocity component in the direction orthogonal to the moment variable is tangentially discontinuous (normal discontinuities are well handled by flux conditions). This is quite a generic set-up which is encountered in many practical situations but usually handled theoretically by regularizing the discontinuity.

We will show in the next paragraph that, for arbitrary velocity fields $g_{f}(t, x, y)$, the reduced model leads to measure valued solutions in finite time and we will propose in the sequel a specific treatment to cope with this difficulty.

2.3. Obtention of moment equations. In this paragraph we derive formally two 1-dimensional PDEs for the zeroth and first order moment of the solution with respect to $y$

$$
\left\{\begin{array}{l}
\widetilde{\rho}(t, x)=\int_{\mathbb{R}^{+}} \Phi(t, x, y) \mathrm{d} y \\
\widetilde{M}(t, x)=\int_{\mathbb{R}^{+}} y \Phi(t, x, y) \mathrm{d} y .
\end{array}\right.
$$

which we name respectively the mass and maturity distributions in $x$. Since this operation is performed scalarwise, we temporarily drop the $f$ index to alleviate the notations. We integrate the 2D PDE (2.10) over $y$

$$
\begin{aligned}
-\int_{\mathbb{R}^{+}} \Lambda(t, x, y) & \Phi(t, x, y) \mathrm{d} y=\int_{\mathbb{R}^{+}}\left(\partial_{t} \Phi+\partial_{x}(g \Phi)+\partial_{y}(h \Phi)\right) \mathrm{d} y \\
= & \int_{\mathbb{R}^{+}}\left(\partial_{t} \Phi+\partial_{x}(g \Phi)\right) \mathrm{d} y+\int_{0}^{y_{s}} \partial_{y}(h \Phi) \mathrm{d} y+\int_{y_{s}}^{+\infty} \partial_{y}(h \Phi) \mathrm{d} y \\
= & \partial_{t} \int_{\mathbb{R}^{+}} \Phi \mathrm{d} y+\partial_{x} \int_{\mathbb{R}^{+}} g(t, x, y) \Phi \mathrm{d} y+[h(t, x, y) \Phi]_{0}^{y_{s}}+[h(t, x, y) \Phi]_{y_{s}}^{+\infty},
\end{aligned}
$$

from which we obtain, using boundary conditions (2.15-2.16),

$$
\int_{\mathbb{R}^{+}} \Lambda(t, x, y) \Phi(t, x, y) \mathrm{d} y=\partial_{t} \widetilde{\rho}(t, x)+\partial_{x} \int_{\mathbb{R}^{+}} g(t, x, y) \Phi \mathrm{d} y .
$$

Then we multiply (2.10) by $y$ and integrate it over $y$

$$
\begin{aligned}
& -\int_{\mathbb{R}^{+}} y \Lambda(t, x, y) \Phi \mathrm{d} y=\int_{\mathbb{R}^{+}}\left(y \partial_{t} \Phi+\partial_{x}(y g \Phi)+y \partial_{y}(h \Phi)\right) \mathrm{d} y \\
= & \partial_{t} \int_{\mathbb{R}^{+}} y \Phi \mathrm{d} y+\partial_{x} \int_{\mathbb{R}^{+}} y g(t, x, y) \Phi \mathrm{d} y+[y h \Phi]_{0}^{y_{s}}+[y h \Phi]_{y_{s}}^{+\infty}-\int_{\mathbb{R}^{+}} h \Phi \mathrm{d} y,
\end{aligned}
$$

which boils down, again using boundary conditions (2.15-2.16), to

$$
-\int_{\mathbb{R}^{+}} y \Lambda(t, x, y) \Phi \mathrm{d} y=\partial_{t} \int_{\mathbb{R}^{+}} y \Phi \mathrm{d} y+\partial_{x} \int_{\mathbb{R}^{+}} y g(t, x, y) \Phi \mathrm{d} y-\int_{\mathbb{R}^{+}} h \Phi \mathrm{d} y .
$$

Generalizing the method described in Section 2.1, let us assume $\widetilde{\rho}(t, x)>0$, and define

$$
\zeta(t, x)=\frac{\widetilde{M}(t, x)}{\widetilde{\rho}(t, x)},
$$

and use as a candidate solution

$$
\Phi(t, x, y)=\widetilde{\rho}(t, x) \delta(y-\zeta(t, x)),
$$


which, plugged into (2.20) and (2.21), yields

$$
\left\{\begin{aligned}
& \partial_{t} \widetilde{\rho}(t, x)+\partial_{x}(g(t, x, \zeta(t, x)) \widetilde{\rho}(t, x))=-\Lambda(t, x, \zeta) \widetilde{\rho}(t, x) \\
& \partial_{t}(\zeta(t, x) \widetilde{\rho}(t, x))+\partial_{x}(\zeta(t, x) g(t, x, \zeta(t, x)) \widetilde{\rho}(t, x)) \\
&=(h(t, x, \zeta(t, x))-\zeta \Lambda(t, x, \zeta)) \widetilde{\rho}(t, x)
\end{aligned}\right.
$$

We intend to show that even when $g(t, x, y)$ varies smoothly in $y$, system $(2.23)$ admits in general solutions that are measures rather than functions. Restricting for the sake of simplicity to $g(t, x, y)=g(y), h=0$ and $\Lambda=0,(2.23)$ boils down to

$$
\left\{\begin{array}{l}
\partial_{t} \widetilde{\rho}(t, x)+\partial_{x}(g(\zeta(t, x)) \widetilde{\rho}(t, x))=0 \\
\partial_{t}(\zeta(t, x) \widetilde{\rho}(t, x))+\partial_{x}(\zeta(t, x) g(\zeta(t, x)) \widetilde{\rho}(t, x))=0 .
\end{array}\right.
$$

Let us now examine the hyperbolic properties of this $2 \times 2$ nonlinear first order system over its natural phase space

$$
\mathcal{V}=\left\{(\widetilde{\rho}, \widetilde{\rho} \zeta) \in \mathbb{R}^{2}, \widetilde{\rho}>0, \widetilde{\rho} \zeta \in \mathbb{R}\right\} .
$$

To this end, let us rewrite (2.24) in the non conservative form,

$$
\begin{aligned}
& \partial_{t} \widetilde{\rho}(t, x)+g(\zeta(t, x)) \partial_{x} \widetilde{\rho}(t, x)+g^{\prime}(\zeta(t, x)) \widetilde{\rho}(t, x) \partial_{x} \zeta(t, x)=0, \\
\widetilde{\rho}(t, x) \partial_{t} \zeta(t, x)+\underline{\zeta(t, x) \partial_{t} \tilde{\rho}(t, x)}+g(\zeta(t, x)) \widetilde{\rho}(t, x) \partial_{x} \zeta(t, x) & \\
+\zeta(t, x) g^{\prime}(\zeta(t, x)) \widetilde{\rho}(t, x) \partial_{x} \zeta(t, x)+\zeta(t, x) g(\zeta(t, x)) \partial_{x} \tilde{\rho}(t, x) & =0,
\end{aligned}
$$

where we assume that both $\widetilde{\rho}$ and $\zeta$ are smooth. In (2.26) we can cancel three terms corresponding to equation (2.25) multiplied by $\zeta$. We obtain finally

$$
\left\{\begin{array}{l}
\partial_{t} \widetilde{\rho}+g(\zeta) \partial_{x} \widetilde{\rho}+g^{\prime}(\zeta) \widetilde{\rho} \partial_{x} \zeta=0 \\
\partial_{t} \zeta+g(\zeta) \partial_{x} \zeta=0
\end{array}\right.
$$

for which the Jacobian matrix (in $\widetilde{\rho}$ and $\zeta$ ) reads

$$
J=\left(\begin{array}{cc}
g(\zeta) & g^{\prime}(\zeta) \widetilde{\rho} \\
0 & g(\zeta)
\end{array}\right)
$$

which is clearly non diagonalizable (weakly hyperbolic), with a double eigenvalue $g(\zeta)$. A well known difficulty with weakly hyperbolic systems stems out from the property that the solution may become a measure [20], and we will now show that this undesirable feature is precisely met in our framework.

We consider a smooth initial data with $\zeta_{0}(x)$ taking values in a strictly monotonous region of $g(\zeta)$ so that $g^{\prime}\left(\zeta_{0}(x)\right) \frac{\mathrm{d} \zeta_{0}}{\mathrm{~d} x}<0$. Under this assumption, the method of characteristics applied to the scalar PDE governing $\zeta$ implies that the smoothness of the initial data is not preserved by the solution $\zeta(t, x)$, which develops a discontinuity at a finite time $T^{\star}([17]$, page 27$)$ :

$$
0<T^{\star}:=-\frac{1}{\min g^{\prime}\left(\zeta_{0}(x)\right) \frac{\mathrm{d} \zeta_{0}}{\mathrm{~d} x}}<\infty,
$$

at which at least one pair of characteristics intersects. After $T^{\star}$ the non conservative form (2.27) is no longer valid, hence we are led to revert to the conservative form 
of PDEs in order to investigate the possibility of solutions which are only piecewise continuous. Along the surfaces of discontinuity which propagate at speed $\sigma$, the solution should satisfy the classical Rankine Hugoniot jump condition with left and right states $\left(\widetilde{\rho}_{-}, \zeta_{-}\right)$and $\left(\widetilde{\rho}_{+}, \zeta_{+}\right)$. From $(2.24)$ we should have

$$
\begin{aligned}
-\sigma\left(\widetilde{\rho}_{+}-\widetilde{\rho}_{-}\right)+\left(\widetilde{\rho}^{+} g\left(\zeta^{+}\right)-\widetilde{\rho}^{-} g\left(\zeta^{-}\right)\right) & =0 \\
-\sigma\left(\widetilde{\rho}_{+} \zeta_{+}-\widetilde{\rho}_{-} \zeta_{-}\right)+\left(\widetilde{\rho}_{+} \zeta_{+} g\left(\zeta_{+}\right)-\widetilde{\rho}_{-} \zeta_{-} g\left(\zeta_{-}\right)\right) & =0 .
\end{aligned}
$$

with

$$
g\left(\zeta_{+}\right)<g\left(\zeta_{-}\right) \text {and } \widetilde{\rho}_{-}>0, \widetilde{\rho}_{+}>0
$$

from which we define

$$
D=\widetilde{\rho}^{+}\left(g\left(\zeta^{+}\right)-\sigma\right)=\widetilde{\rho}^{-}\left(g\left(\zeta^{-}\right)-\sigma\right) .
$$

Then the second equation in (2.24) leads to

$$
-\sigma\left(\left(\widetilde{\rho}^{+} \zeta^{+}\right)-\left(\widetilde{\rho}^{-} \zeta^{-}\right)\right)+\left(\widetilde{\rho}^{+} g\left(\zeta^{+}\right) \zeta^{+}-\widetilde{\rho}^{-} g\left(\zeta^{-}\right) \zeta^{-}\right)=D\left(\zeta^{+}-\zeta^{-}\right)=0 .
$$

If $D \neq 0$ then $\zeta^{+}=\zeta^{-}$, which contradicts (2.28) therefore $D=0$ : discontinuous solutions cannot exist. If $\zeta$ is discontinuous then $\widetilde{\rho}$ becomes a measure (as may occur for zero pressure gas [6]) in finite time. This will indeed happen since $\zeta$ develops discontinuities in finite time when its initial data is decreasing in $x$.

2.4. Alternative 2D set-up. In [26], a reduced model has been derived for a slightly different 2D model. The maturation velocity satisfies hypothesis (2.4), the cell cycle in the proliferation zone is no more subdivided into two phases $G 1$ and $S M$ and the model coefficients are assumed to be smooth, thanks to a regularization across the internal boundary between the cell cycle and differentiation domain, as shown in Figure 2.2. After reducing the 2D model to a 1D model, an additional simplification is proposed in [26], which consists in replacing the doubling flux condition modeling mitosis by a source term distributed over the whole cell cycle. This simplification makes it possible to further reduce the model into an ODE system for the macroscopic mass and maturity depending on time only. In the present work, we want to preserve the modeling of the localized mitosis, as well as the cell cycle phases, since they are important features in cell biology. However, we have shown in the previous paragraph that, in the general case with discontinuous velocity coefficients, the technique proposed in [26], if applied directly to the original 2D model, leads to an ill-posed 1D system. We propose to circumvent this obstacle by introducing a slightly modified 2D multiscale model, which is equivalent to the original one in terms of moments and is suited to reduction through averaging over $y$. 

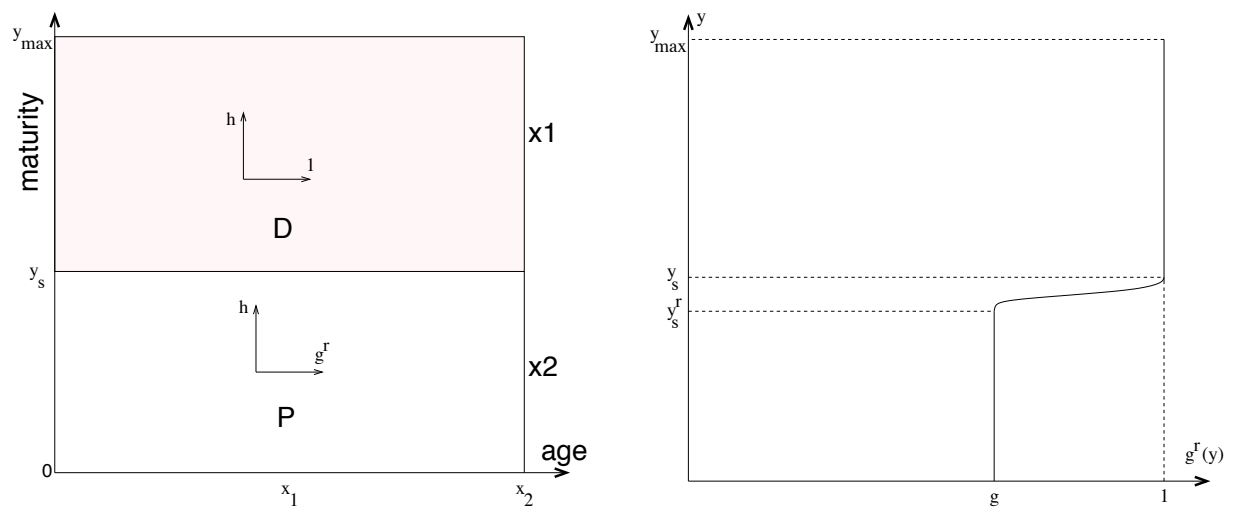

FIG. 2.2. Simplified model studied in [26]. Left panel: computing domain $\Omega$ without cell cycle subdivisions. Right panel: regularized horizontal (aging) velocity $g^{r}(t, y)$ used in [26] displayed as a function of $y$. The variable $y$ is displayed vertically, as in the left panel, to emphasize the regularization between $y_{s}^{r}$ and $y_{s}$.

As shown in the right panel of Figure 2.1, we now define the PDE system (2.10) for $(x, y) \in \Omega=G 1 \cup S M \cup D 1 \cup D 2$ where

$$
\left\{\begin{array}{l}
G 1=\left\{(x, y), 0<x<x_{1}, 0<y<y_{s}\right\} \\
S M=\left\{(x, y), x_{1}<x<x_{2}, 0<y<y_{s}\right\} \\
D 1=\left\{(x, y), 0<x<x_{1}, y_{s}<y\right\} \\
D 2=\left\{(x, y), x_{1}<x<x_{2}, y_{s}<y\right\}
\end{array}\right.
$$

The horizontal velocity $g_{f}$ is now continuous across the boundary between $G_{1}$ and $D$ but discontinuous across the boundary between $D_{1}$ and $D_{2}$, which leads to the boundary condition

$$
g_{f}\left(t, 0^{+}, y\right) \Phi_{f}\left(t, 0^{+}, y\right)=\Phi_{f}\left(t, x_{2}^{-}, y\right), \quad \forall y>y_{s},
$$

instead of (2.14) and condition (2.12) is extended on the whole maturity range

$$
\Phi_{f}\left(t, x_{1}^{+}, y\right)=g_{f}\left(t, x_{1}^{-}, y\right) \Phi_{f}\left(t, x_{1}^{-}, y\right), \quad \forall y, 0<y
$$

on the new internal boundary between $D_{1}$ and $D_{2}$.

The only difference with the original set-up lies in the differentiation phase. From the modeling viewpoint the notion of cell cycle does not hold in this part of the domain where the cells do not divide anymore, which is why the age variable matches time $t$ in $D$ in the original model (2.36). The age rather becomes a measure of senescence in the long term. However we will show in the remaining of this paragraph that the macroscopic outputs of the two set-ups are equivalent, at least for practical purposes such as parameter identification.

2.4.1. Theoretical result in the case of an affine maturation velocity. We first show that if the maturation velocity is an affine function of $y$, and in the absence of cell death, the two 2D set-ups are equivalent in terms of the zeroth and first order moments. Without loss of generality, we restrict our study to the scalar case of a single population. We denote by $\Phi^{(1)}$ the solution of the original set-up (2.10)-(2.17) and $\Phi^{(2)}$ the solution of the modified set-up (2.10),(2.13),(2.15),(2.17), (2.30)-(2.32). 
TheOREM 2.3. Denoting by $\rho^{(i)}$ and $M^{(i)} \quad(i=1,2)$ the zeroth and the first moments (2.19) of the solutions $\Phi^{(i)}, i=1,2$

$$
\left\{\begin{array}{l}
\rho^{(i)}(t)=\int_{0}^{x_{2}} \int_{\mathbb{R}^{+}} \Phi^{(i)}(t, x, y) d x d y \\
M^{(i)}(t)=\int_{0}^{x_{2}} \int_{\mathbb{R}^{+}} y \Phi^{(i)}(t, x, y) d x d y
\end{array}\right.
$$

in the absence of source term $(\Lambda=0)$, we have

$$
\rho^{(1)}(t)=\rho^{(2)}(t), \quad \forall t>0,
$$

and, if $h(t, x, y)=c_{1}(t) y+c_{2}(t)$, we have

$$
M^{(1)}(t)=M^{(2)}(t), \quad \forall t>0 .
$$

Proof. For any $t$, we have that

$$
\frac{d}{\mathrm{~d} t} \rho^{(1)}(t)=\frac{d}{\mathrm{~d} t} \int_{0}^{x_{2}} \int_{\mathbb{R}^{+}} \Phi^{(1)}(t, x, y) \mathrm{d} x \mathrm{~d} y=\int_{0}^{x_{2}} \int_{\mathbb{R}^{+}} \frac{\partial}{\partial t} \Phi^{(1)}(t, x, y) \mathrm{d} x \mathrm{~d} y .
$$

We split the computing domain into the three $D, G 1$ and $S M$ zones, where the solution is continuous, and use the PDE (2.10) to obtain

$$
\begin{array}{rl}
\frac{d}{\mathrm{~d} t} \rho^{(1)}(t)=\int_{0}^{x_{1}} \int_{0}^{y_{s}} \frac{\partial}{\partial t} \Phi^{(1)}(t, x, y) \mathrm{d} & x \mathrm{~d} y+\int_{x_{1}}^{x_{2}} \int_{0}^{y_{s}} \frac{\partial}{\partial t} \Phi^{(1)}(t, x, y) \mathrm{d} x \mathrm{~d} y \\
& +\int_{0}^{x_{2}} \int_{y_{s}}^{+\infty} \frac{\partial}{\partial t} \Phi^{(1)}(t, x, y) \mathrm{d} x \mathrm{~d} y .
\end{array}
$$

Using the periodic boundary condition (2.14), the doubling condition (2.13), the transmission conditions $(2.12,2.15)$, the waterproof condition (2.16) and the null conditions on the outer horizontal bounds (2.18), we obtain

$$
\begin{aligned}
& \frac{d}{\mathrm{~d} t} \rho^{(1)}(t)=-\int_{0}^{y_{s}} \Phi^{(1)}\left(t, x_{2}^{-}, y\right) \mathrm{d} y+\int_{0}^{y_{s}} \Phi^{(1)}\left(t, x_{1}^{+}, y\right) \mathrm{d} y-\int_{y_{s}}^{+\infty} \Phi^{(1)}\left(t, x_{2}^{-}, y\right) \mathrm{d} y \\
& +\int_{y_{s}}^{+\infty} \Phi^{(1)}\left(t, 0^{+}, y\right) \mathrm{d} y-\int_{0}^{x_{2}} h(t, x,+\infty) \Phi^{(1)}(t, x,+\infty) \mathrm{d} x+\int_{0}^{x_{2}} h\left(t, x, y_{s}^{+}\right) \Phi^{(1)}\left(t, x, y_{s}^{+}\right) \mathrm{d} x \\
= & \int_{0}^{y_{s}} \Phi^{(1)}\left(t, x_{2}^{-}, y\right) \mathrm{d} y .
\end{aligned}
$$

Similarly, using the modified set-up (2.30), with the additional transmission condition (2.32) and the periodic condition (2.31) instead of (2.14) we show that

$$
\frac{d}{\mathrm{~d} t} \rho^{(2)}(t)=\int_{0}^{y_{s}} \Phi^{(2)}\left(t, x_{2}^{-}, y\right) \mathrm{d} y
$$

When $y \leq y_{s}$, since $\Phi^{(1)}$ and $\Phi^{(2)}$ satisfy the same PDE (2.10) with the same initial data (2.17), and since the velocities $g$ and $h$ are positive, we get

$$
\Phi^{(1)}(t, x, y)=\Phi^{(2)}(t, x, y) \text { for } 0 \leq x \leq x_{2}, 0 \leq y \leq y_{s},
$$


and consequently

$$
\int_{0}^{y_{s}} \Phi^{(1)}\left(t, x_{2}^{-}, y\right) \mathrm{d} y=\int_{0}^{y_{s}} \Phi^{(2)}\left(t, x_{2}^{-}, y\right) \mathrm{d} y
$$

Therefore

$$
\frac{d}{\mathrm{~d} t} \rho^{(1)}(t)=\frac{d}{\mathrm{~d} t} \rho^{(2)}(t), \quad \forall t>0
$$

Moreover, we have that

$$
\begin{aligned}
\rho^{(1)}(0) & =\int_{0}^{x_{2}} \int_{\mathbb{R}^{+}} \Phi^{(1)}(0, x, y) \mathrm{d} x \mathrm{~d} y \\
& =\int_{0}^{x_{2}} \int_{\mathbb{R}^{+}} \Phi_{0}(x, y) \mathrm{d} x \mathrm{~d} y=\int_{0}^{x_{2}} \int_{\mathbb{R}^{+}} \Phi^{(2)}(0, x, y) \mathrm{d} x \mathrm{~d} y=\rho^{(2)}(0)
\end{aligned}
$$

Thus, we get

$$
\rho^{(1)}(t)=\rho^{(2)}(t), \quad \forall t>0 .
$$

Applying the same reasoning to the first moment, we have, using (2.10), that

$$
\begin{aligned}
\frac{d}{\mathrm{~d} t} M^{(1)}(t)= & \frac{d}{\mathrm{~d} t} \int_{0}^{x_{2}} \int_{\mathbb{R}^{+}} y \Phi^{(1)}(t, x, y) \mathrm{d} x \mathrm{~d} y=\int_{0}^{x_{2}} \int_{\mathbb{R}^{+}} y \frac{\partial}{\partial t} \Phi^{(1)}(t, x, y) \mathrm{d} x \mathrm{~d} y \\
= & -\int_{0}^{x_{1}} \int_{0}^{y_{s}} y\left[\frac{\partial g(t, x, y) \Phi^{(1)}(t, x, y)}{\partial x}+\frac{\partial h(t, x, y) \Phi^{(1)}(t, x, y)}{\partial y}\right] \mathrm{d} x \mathrm{~d} y \\
& -\int_{x_{1}}^{x_{2}} \int_{0}^{y_{s}} y \frac{\partial}{\partial x} \Phi^{(1)}(t, x, y) \mathrm{d} x \mathrm{~d} y \\
& -\int_{0}^{x_{2}} \int_{y_{s}}^{+\infty} y\left[\frac{\partial}{\partial x} \Phi^{(1)}(t, x, y)+\frac{\partial}{\partial y} h(t, x, y) \Phi^{(1)}(t, x, y)\right] \mathrm{d} x \mathrm{~d} y .
\end{aligned}
$$

Integrating by parts and using the outer and inner boundary conditions we have

$$
\frac{d}{\mathrm{~d} t} M^{(1)}(t)=\int_{0}^{y_{s}} y \Phi^{(1)}\left(t, x_{2}^{-}, y\right) \mathrm{d} y+\int_{\mathbb{R}^{+}} \int_{0}^{x_{2}} h(t, x, y) \Phi^{(1)}(t, x, y) \mathrm{d} x \mathrm{~d} y .
$$

On the other hand, similar computation for the modified set-up boils down to

$$
\frac{d}{\mathrm{~d} t} M^{(2)}(t)=\int_{0}^{y_{s}} y \Phi^{(2)}\left(t, x_{2}^{-}, y\right) \mathrm{d} y+\int_{\mathbb{R}^{+}} \int_{0}^{x_{2}} h(t, x, y) \Phi^{(2)}(t, x, y) \mathrm{d} x \mathrm{~d} y .
$$

If $h(t, x, y)=c_{1}(t) y+c_{2}(t)$ and $\Lambda=0$, we have that for $i=1,2$

$$
\begin{aligned}
\frac{d}{\mathrm{~d} t} M^{(i)}(t) & =\int_{0}^{y_{s}} y \Phi^{(i)}\left(t, x_{2}^{-}, y\right) \mathrm{d} y+\int_{0}^{x_{2}} \int_{\mathbb{R}^{+}} h(t, x, y) \Phi^{(i)}(t, x, y) \mathrm{d} x \mathrm{~d} y \\
& =\int_{0}^{y_{s}} y \Phi^{(i)}\left(t, x_{2}^{-}, y\right) \mathrm{d} y+\int_{0}^{x_{2}} \int_{\mathbb{R}^{+}}\left(c_{1}(t) y+c_{2}(t)\right) \Phi^{(i)}(t, x, y) \mathrm{d} x \mathrm{~d} y \\
& =\int_{0}^{y_{s}} y \Phi^{(i)}\left(t, x_{2}^{-}, y\right) \mathrm{d} y+c_{1}(t) M^{(i)}(t)+c_{2}(t) \rho^{(i)}(t) .
\end{aligned}
$$


Using (2.34) again, we obtain

$$
\int_{0}^{y_{s}} y \Phi^{(1)}\left(t, x_{2}^{-}, y\right) \mathrm{d} y=\int_{0}^{y_{s}} y \Phi^{(2)}\left(t, x_{2}^{-}, y\right) \mathrm{d} y .
$$

Furthermore, since $\rho^{(1)}(t)=\rho^{(2)}(t)$, we get

$$
\frac{d}{\mathrm{~d} t} M^{(1)}(t)-c_{1}(t) M^{(1)}(t)=\frac{d}{\mathrm{~d} t} M^{(2)}(t)-c_{1}(t) M^{(2)}(t),
$$

which is equivalent to

$$
\frac{d}{\mathrm{~d} t}\left[M^{(1)}(t)-M^{(2)}(t)\right]=+c_{1}(t)\left[M^{(1)}(t)-M^{(2)}(t)\right],
$$

from which we obtain

$$
M^{(1)}(t)-M^{(2)}(t)=e^{\int_{0}^{t} c_{1}(s) d s}\left[M^{(1)}(0)-M^{(2)}(0)\right]=0, \quad \forall t \geq 0 .
$$

This complete the proof of the theorem.

2.4.2. Numerical simulations in a non affine case. We now turn to a more specific set-up arising in the modeling of the development of cell populations within terminally developing ovarian follicles [16]. Ovarian follicles are the basic anatomical and functional units of the ovaries sheltering the oocyte. In the latest stages, ovarian follicles participate in entangled hormonal feedback loops involving the pituitary gland (and hypothalamus), so that their fates are interdependent. The interaction amongst a cohort of $F$ follicles that enter simultaneously the final stage of growth before ovulation is modeled by system (2.10) whose unknown is the vector of granulosa cell densities $\Phi=\left(\phi_{f}\right)_{f=1, \ldots, F}$. The age variable, and the associated age velocity $g_{f}$, account for possible time dependent durations of phase G1, due to the hormonal control exerted by pituitary hormones (mainly the follicle-stimulating-hormone, FSH) whose local availability may vary differentially from one follicle to the other. The aging function $g_{f}$ is defined by

$$
g_{f}(t, x, y)= \begin{cases}g_{1}^{f} u_{f}(t)+g_{2}^{f} & \text { for } \quad(x, y) \in G 1 \\ 1 & \text { for } \quad(x, y) \in S M \cup D,\end{cases}
$$

where $g_{1}^{f}, g_{2}^{f}$ are real positive constants. The control $u_{f}(t)$ is the locally bioavailable FSH level

$$
u_{f}(t)=\left(b_{1}^{f}+\frac{1-b_{1}^{f}}{1+e^{b_{2}^{f}\left(M_{f}(t)-b_{3}^{f}\right)}}\right) U(t),
$$

where $b_{1}^{f}, b_{2}^{f}$ and $b_{3}^{f}$ are real positive constants and $U(t)$ is the global FSH resource

$$
U(t)=U_{\min }+\frac{U_{\max }-U_{\min }}{1+e^{c\left(M_{T}(t)-m\right)}},
$$

with $U_{\min }, c$ and $m$ real positive constants.

In (2.37) and (2.38), $M_{f}(t)$ and $M_{T}(t)$ are respectively the mean maturity of follicle $f$

$$
M_{f}(t)=\iint_{\Omega} y \Phi_{f}(t, x, y) \mathrm{d} x \mathrm{~d} y
$$


and the ovarian maturity, measuring the contribution of all follicles,

$$
M_{T}(t)=\sum_{f=1}^{N_{f}} M_{f}(t),
$$

The same feedback control, which couples the behaviors of the follicles in a non local manner, appears in the definition of the maturity function $h_{f}$

$$
h_{f}(t, x, y)= \begin{cases}\tau_{h}^{f}\left[-y^{2}+\left(c_{1}^{f} y+c_{2}^{f}\right)\left(1-e^{-\frac{u_{f}(t)}{\bar{u}^{f}}}\right)\right] & \text { for }(x, y) \in G 1 \cup D \\ 0 & \text { for }(x, y) \in S M\end{cases}
$$

where $c_{1}^{f}, c_{2}^{f}, \tau_{h}^{f}$ and $\bar{u}^{f}$ are real positive constants, and in the source term, that represents cell death through apoptosis

$$
\Lambda(t, x, y)= \begin{cases}K e^{-\left(\frac{y-y_{s}}{\bar{y}^{2}}\right)^{2}} \frac{U_{\max }-U(t)}{U_{\max }} \mathbf{1}_{y_{s}^{-}<y<y_{s}^{+}} & \text {for }(x, y) \in G 1 \cup D \\ 0 & \text { for }(x, y) \in S M\end{cases}
$$

where $K$ and $\bar{y}$ are real positive constants.

To alleviate the notations, we will drop in the sequel the $f$ upper index indicating that constants $g_{1}^{f}, g_{2}^{f}, c_{1}^{f}, c_{2}^{f}, \tau_{h}^{f}, \bar{u}^{f}, b_{1}^{f}, b_{2}^{f}$ and $b_{3}^{f}$ may vary among follicles, and we will also drop the $f$ suffix in the single population case.

When integrating PDE (2.10) to compute the first order moment equation, the $-y^{2}$ contribution in the maturation velocity $h(t, x, y)(2.41)$, and the specific form of the cell death rate (2.42) lead to a recursive relation involving moments of three consecutive orders instead of simply two as in (2.35). Postponing the theoretical study to future work, we check that for realistic parameter values, the zeroth and the first order moments are numerically comparable. We refer the reader to [2, 4] for the description and validation of the $2 \mathrm{D}$ numerical method used to perform the simulations. The density for both set-ups is computed using a second order Finite Volume scheme on multiresolution-driven adaptive meshes with the parameter values provided in Table 5.1. The initial condition is piecewise exponential

$$
\psi_{0}(x)= \begin{cases}\frac{1}{(2 g)^{x}} & \text { for } 0 \leq x<1 / 2 \\ \left(\frac{g}{2}\right)^{x} & \text { for } 1 / 2 \leq x<1\end{cases}
$$

where $g$ is the initial value of the aging speed (2.36). The results of these simulations on the microscopic level are displayed in Figure 2.3 as snapshots of the density $\phi(t, x, y)$ at two different times $t=4.37$ and $t=5$. In the original set-up (left panels) the solution is continuous in the differentiation domain (above $y_{s}$ ). In the modified setup (right panels), the sharp change in color at $x=x_{1}=0.5$ indicates that the discontinuity, biologically relevant for the cell division cycle (below $y_{s}$ ), is artificially replicated in the differentiation subdomain. In the top panels, at time $t=4.37$, the support of the density lies on both sides of the inner boundary $y=y_{s}$. At time $t=5$, all cells have exited the cell cycle. As predicted and proved above, the 
numerical densities computed for the two set-ups are identical in the bottom part of the computational domain $G_{1} \cup S M$, yet they are different above. Nevertheless, at each time step, the total cell number and the maturity are the same for both set-ups.

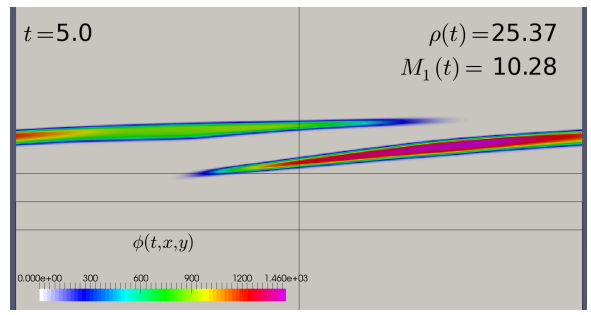

a) Original set-up at time $t=4.37$

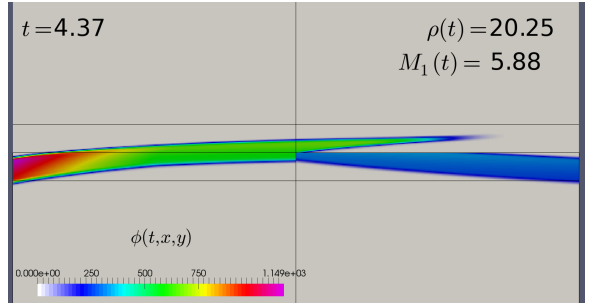

c) Original set-up at time $t=5$.

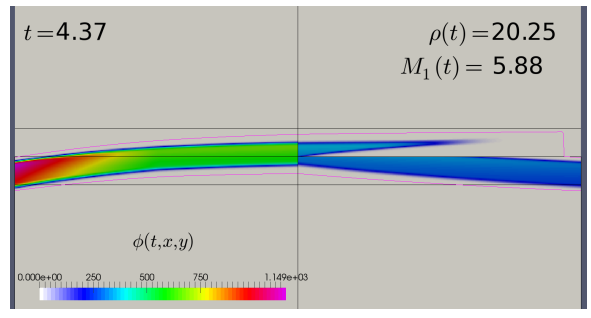

b) Modified set-up at time $t=4.37$

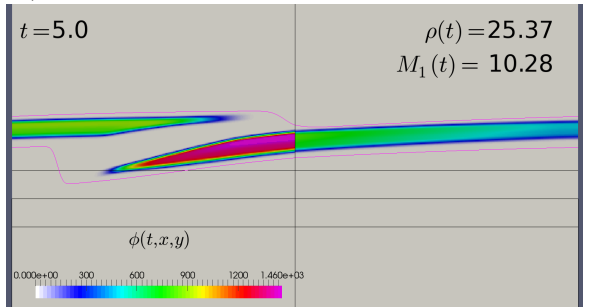

d) Modified set-up at time $t=5$.

Fig. 2.3. Comparison between the original (left panels) and modified (right panels) set-ups on the microscopic level. The values of the simulation parameters are gathered in Table 5.1. The top panels display the cell density at time 4.37 when the density cloud crosses the interface between the proliferation and differentiation subdomains. The bottom panels display the cell density at time $t=5$., where $\operatorname{Supp}(\phi(t,).) \subset D$. The horizontal axis is age $x$ and vertical axis maturity $y$, the vertical black line separates phases $G 1$ and $S M$ at $x=0.5$. The same color code is used for both set-ups but varies with time. The horizontal black lines indicate the maturity thresholds $y_{s}^{-}=0.25, y_{s}=0.3$ and $y_{s}^{+}=0.35$. The magenta curves surrounding the density in the right panels correspond to the maturity component $\zeta(t, x)$ of the reduced model (see paragraph 5.1 for comment).

3. Properties of the reduced model. In this part, we show that for the modified 2D set-up introduced in section 2.4, where the horizontal velocity $g(t, x)$ does not depend on the vertical variable $y$, the reduced model (2.23), when endowed with adequate initial and boundary conditions, can be used to define a weak solution of the full model, starting from an initial Dirac mass in maturity. We also prove the convergence in long time of the 2D solution starting from a compactly supported initial condition towards a Dirac in maturity distribution.

3.1. Link with the 2D model. Using the same method as in Paragraph 2.3, we obtain 1D PDEs for the zeroth and first moments. Now the aging velocity $g$ depends only on $t$ and $x$ and not anymore on $\zeta$ as in system (2.23); therefore we do not risk to develop measure-valued solutions in finite time, as it was the case in the original set-up, and we can prove the following theorem:

Theorem 3.1. Let $\zeta(t, x)$ be the solution of

$$
\frac{\partial}{\partial t} \zeta(t, x)+g(t, x) \frac{\partial}{\partial x} \zeta(t, x)=h(t, x, \zeta(t, x)),
$$

with the initial data

$$
\zeta(0, x)=\zeta^{0}(x), \quad \zeta_{0} \in C^{1}\left(\left[0, x_{2}\right]\right), x_{2}-\text { periodic },
$$


and the boundary conditions

$$
\left\{\begin{array}{l}
\zeta\left(t, x_{1}^{-}\right)=\zeta\left(t, x_{1}^{+}\right) \quad \text { almost everywhere, } \\
\zeta(t, 0)=\zeta\left(t, x_{2}\right)
\end{array}\right.
$$

Let $\widetilde{\rho}(t, x)$ be the solution of

$$
\frac{\partial}{\partial t} \widetilde{\rho}(t, x)+\frac{\partial}{\partial x}(g(t, x) \widetilde{\rho}(t, x))=-\Lambda(t, x, \zeta(t, x)) \widetilde{\rho}(t, x),
$$

with the boundary conditions

$$
\left\{\begin{array}{l}
g\left(t, 0^{+}\right) \widetilde{\rho}\left(t, 0^{+}\right)=2 \widetilde{\rho}\left(t, x_{2}^{-}\right), \quad \text { if } \zeta\left(t, x_{2}\right)<y_{s} \\
g\left(t, 0^{+}\right) \widetilde{\rho}\left(t, 0^{+}\right)=\widetilde{\rho}\left(t, x_{2}^{-}\right), \quad \text { if } \zeta\left(t, x_{2}\right)>y_{s} \\
g\left(t, x_{1}^{-}\right)\left(t, 0^{+}\right) \widetilde{\rho}\left(t, x_{1}^{-}\right)=\widetilde{\rho}\left(t, x_{1}^{+}\right)
\end{array}\right.
$$

and the initial data

$$
\widetilde{\rho}(0, x)=\rho^{0}(x)>0, \forall x \in\left[0, x_{2}\right]
$$

satisfying (3.4) with $\rho^{0}$ of class $C^{1}$ on $] 0, x_{1}[$ and $] x_{1}, x_{2}[$.

Then $\tilde{\Phi}(t, x, y)=\widetilde{\rho}(t, x) \delta(y-\zeta(t, x))$ is a weak solution to the 2D model (2.10), (2.12),(2.13), (2.31), (2.32) defined on the domain (2.30) for the initial condition $\phi_{0}(x, y)=\rho^{0}(x) \delta\left(y-\zeta_{0}(x)\right)$.

Before proving this theorem we recall the weak formulation of the 2D model:

Lemma 3.2. $\Phi$ is a weak solution of the $2 D$ model (in the distributional sense) if for all $\psi \in C^{\infty}\left(\left[0,+\infty\left[\times\left[0, x_{2}\right] \times \mathbb{R}^{+}\right)\right.\right.$compactly supported in time and $y$ and $x_{2}-$ periodic in $x$

$$
\begin{array}{r}
\int_{0}^{+\infty} \int_{0}^{x_{2}} \int_{\mathbb{R}^{+}} \Phi\left(\partial_{t} \psi+g \partial_{x} \psi+h \partial_{y} \psi\right) d y d x d t=\int_{0}^{+\infty} \int_{0}^{x_{2}} \int_{\mathbb{R}^{+}} \Lambda \Phi \psi d y d x d t \\
-\int_{0}^{x_{2}} \int_{\mathbb{R}^{+}} \Phi_{0}(x, y) \psi(0, x, y) d y d x+\frac{1}{2} \int_{0}^{+\infty} \int_{0}^{y_{s}} g\left(0^{+}, y\right) \Phi\left(t, 0^{+}, y\right) \psi(t, 0, y) d y d t .(3.5)
\end{array}
$$

We postpone the proof of this Lemmato Appendix B.

Proof. We show that $\tilde{\Phi}(t, x, y)$ satisfies (3.5). For all $\psi \in C^{\infty}\left(\left[0,+\infty\left[\times\left[0, x_{2}\right] \times\right.\right.\right.$ $\mathbb{R}^{+}$) compactly supported in time and $y$ and $x_{2}$-periodic in $x$, we have

$$
\begin{aligned}
I & =\int_{0}^{+\infty} \int_{0}^{x_{2}} \int_{\mathbb{R}^{+}} \tilde{\Phi}(t, x, y)\left(\partial_{t} \psi(t, x, y)+g(t, x) \partial_{x} \psi(t, x, y)+h(t, x, y) \partial_{y} \psi(t, x, y)\right) \mathrm{d} y \mathrm{~d} x \mathrm{~d} t \\
& =\int_{0}^{+\infty} \int_{0}^{x_{2}} \int_{\mathbb{R}^{+}} \tilde{\rho}(t, x) \delta(y-\zeta(t, x))\left(\partial_{t} \psi(t, x, y)+g(t, x) \partial_{x} \psi(t, x, y)+h(t, x, y) \partial_{y} \psi(t, x, y)\right) \mathrm{d} y \mathrm{~d} x \mathrm{~d} t \\
& =\int_{0}^{+\infty} \int_{0}^{x_{2}} \widetilde{\rho}(t, x)\left(\partial_{t} \psi(t, x, \zeta(t, x))+g(t, x) \partial_{x} \psi(t, x, \zeta(t, x))+h(t, x, \zeta(t, x)) \partial_{y} \psi(t, x, \zeta(t, x))\right) \mathrm{d} x \mathrm{~d} t
\end{aligned}
$$

We replace $h(t, x, \zeta(t, x))$ by its expression given by (3.1) and reorder the terms to get

$$
\begin{aligned}
I= & \int_{0}^{+\infty} \int_{0}^{x_{2}} \widetilde{\rho}(t, x)\left(\partial_{t} \psi(t, x, \zeta(t, x))+\partial_{t} \zeta(t, x) \partial_{y} \psi(t, x, \zeta(t, x))\right)+ \\
& g(t, x) \widetilde{\rho}(t, x)\left(\partial_{x} \psi(t, x, \zeta(t, x))+\partial_{x} \zeta(t, x) \partial_{y} \psi(t, x, \zeta(t, x))\right) \mathrm{d} x \mathrm{~d} t
\end{aligned}
$$


Denoting $\tilde{\psi}(t, x)=\psi(t, x, \zeta(t, x))$ with the identities

$$
\left\{\begin{array}{l}
\partial_{t} \tilde{\psi}(t, x)=\partial_{t} \psi(t, x, \zeta(t, x))+\partial_{t} \zeta(t, x) \partial_{y} \psi(t, x, \zeta(t, x)) \\
\partial_{x} \tilde{\psi}(t, x)=\partial_{x} \psi(t, x, \zeta(t, x))+\partial_{x} \zeta(t, x) \partial_{y} \psi(t, x, \zeta(t, x))
\end{array}\right.
$$

yields

$$
\begin{aligned}
I= & \int_{0}^{+\infty} \int_{0}^{x_{2}}\left(\widetilde{\rho}(t, x) \partial_{t} \tilde{\psi}(t, x)+g(t, x) \widetilde{\rho}(t, x) \partial_{x} \tilde{\psi}(t, x) \mathrm{d} x \mathrm{~d} t\right. \\
= & \int_{0}^{x_{2}}[\widetilde{\rho}(t, x) \tilde{\psi}(t, x)]_{0}^{+\infty} \mathrm{d} x-\int_{0}^{+\infty} \int_{0}^{x_{2}} \tilde{\psi}(t, x) \partial_{t} \widetilde{\rho}(t, x) \mathrm{d} x \mathrm{~d} t+\int_{0}^{+\infty}[g(t, x) \widetilde{\rho}(t, x) \tilde{\psi}(t, x)]_{0}^{x_{2}} \mathrm{~d} t \\
& -\int_{0}^{+\infty} \int_{0}^{x_{2}} \tilde{\psi}(t, x) \partial_{x}(g(t, x) \widetilde{\rho}(t, x)) \mathrm{d} x \mathrm{~d} t \\
= & -\int_{0}^{x_{2}} \widetilde{\rho}_{0}(x) \tilde{\psi}(0, x) \mathrm{d} x+\int_{0}^{+\infty} \int_{0}^{x_{2}} \Lambda(t, x, \zeta(t, x)) \tilde{\psi}(t, x) \widetilde{\rho}(t, x) \mathrm{d} x \mathrm{~d} t+\int_{0}^{+\infty}[g(t, x) \widetilde{\rho}(t, x) \tilde{\psi}(t, x)]_{0}^{x_{2}} \mathrm{~d} t
\end{aligned}
$$

We define $t_{s}$ such that $\zeta\left(t_{s}, x_{2}\right)=x_{s}$, which is well defined thanks to the positivity of $h$, and we split the last time integral into two

$$
\begin{aligned}
I= & -\int_{0}^{x_{2}} \rho_{0}(x) \tilde{\psi}(0, x) \mathrm{d} x+\int_{0}^{+\infty} \int_{0}^{x_{2}} \Lambda(t, x, \zeta(t, x)) \tilde{\psi}(t, x) \widetilde{\rho}(t, x) \mathrm{d} x \mathrm{~d} t \\
& +\int_{0}^{t_{s}}[g(t, x) \widetilde{\rho}(t, x) \tilde{\psi}(t, x)]_{0}^{x_{2}} \mathrm{~d} t+\int_{t_{s}}^{+\infty}[g(t, x) \widetilde{\rho}(t, x) \tilde{\psi}(t, x)]_{0}^{x_{2}} \mathrm{~d} t .
\end{aligned}
$$

Using boundary conditions $(3.4,3.2)$ and $x$-periodicity of $\psi$ and $\zeta$, the integrand for $t$ between 0 and $t_{s}$ boils down to $\frac{1}{2} g(t, 0) \widetilde{\rho}(t, 0) \tilde{\psi}(t, 0)$ and the integral from $t_{s}$ to $\infty$ vanishes. We can then reintroduce the Dirac dependence in $y$ to obtain (3.5):

$$
\begin{aligned}
I= & -\int_{\mathbb{R}^{+}} \int_{0}^{x_{2}} \tilde{\phi}(0, x, y) \psi(0, x, y) \mathrm{d} x \mathrm{~d} y+\int_{0}^{+\infty} \int_{0}^{y_{s}} \frac{1}{2} g(t, 0) \tilde{\phi}(t, 0, y) \psi(t, 0, y) \mathrm{d} y \mathrm{~d} t \\
& +\int_{0}^{+\infty} \int_{0}^{x_{2}} \int_{\mathbb{R}^{+}} \Lambda(t, x, y) \psi(t, x, y) \tilde{\phi}(t, x, y) \mathrm{d} y \mathrm{~d} x \mathrm{~d} t .
\end{aligned}
$$

Remarks: (i) The boundary conditions in $x(2.12),(2.13)$, and the internal conditions (2.31), (2.32) are well satisfied by the measure solution $\tilde{\phi}$ as long as (3.2) and (3.4) hold. However the condition $(2.16)\left(\phi\left(t, x, y_{s}^{+}\right)=0\right.$ on $\left.S M \cap D_{2}\right)$ is not met by the measure $\tilde{\phi}$, unless an additional condition

$$
\widetilde{\rho}(t, x)=0 \text { for } x \in] x_{1}, x_{2}\left[, \text { if } \zeta(t, x)=y_{s},\right.
$$

is satisfied by $\widetilde{\rho}$ and $\zeta$, which is not compatible in general with the hyperbolic nature of $(3.1,3.3)$.

(ii) We have shown in Paragraph 2.3 that the same derivation for the original setup leads to an unsuitable system of 1D PDEs. Indeed writing (2.23) as a system in $\widetilde{\rho}$ and $\zeta$ yields the boundary conditions

$$
\begin{aligned}
g\left(t, x_{1}^{-}\right) \widetilde{\rho}\left(t, x_{1}^{-}\right) & =\widetilde{\rho}\left(t, x_{1}^{+}\right), \text {if } \zeta\left(t, x_{1}\right)<y_{s}, \\
\widetilde{\rho}\left(t, x_{1}^{-}\right) & =\widetilde{\rho}\left(t, x_{1}^{+}\right), \text {if } \zeta\left(t, x_{1}\right)>y_{s},
\end{aligned}
$$

which, enforced in the numerical scheme to compute the approximate solution, does not produce a good estimate of the original 2D solution. 
3.2. Asymptotic time convergence in maturity. Theorem 2.1 in [26] shows that, in long time, the cell population gets organized and converges in maturity towards the positive root of the maturity velocity. In our model, $g, h$ and the source term $\Lambda$ are discontinuous. Moreover, we do not make, in contrast to [26], the simplifying assumption that there exists $\mu>0$ such that

$$
\frac{\partial}{\partial y} h \leq-\mu \text {. }
$$

Yet, we can still show that the model specified in paragraph 2.4.2 has the long time behavior stated in the following theorem

THEOREM 3.3. Let $\Phi$ be the solution to the system (2.10) with the initial data $\Phi(t=0, x, y)$ positive and satisfying

$$
\operatorname{Supp}(\Phi(t=0, ., .)) \subset\left[0, x_{2}\right] \times\left[y_{\min }^{0}, y_{\max }^{0}\right] \text {, with } 0<y_{\min }^{0}<y_{\max }^{0}<y_{s} .
$$

Then there exist $\eta>0, C>0,0<t_{s}<\infty, 0<y_{0}^{s}<y_{1}^{s}<y_{\max }$, such that if $\zeta_{0}^{s}$ and $\zeta_{1}^{s}$ are the solutions to (3.1) with the initial data at time $t_{s}$

$$
\zeta_{i}^{s}\left(t_{s}, x\right)=y_{i}^{s}, \quad i=0,1,
$$

then, $\Phi$ satisfies

$$
\operatorname{Supp}(\Phi(t, . . .)) \subset\left\{(x, y), x \in\left[0, x_{2}\right], y \in\left[\zeta_{0}^{s}(t, x), \zeta_{1}^{s}(t, x)\right]\right\}, \quad \forall t \geq t_{s},
$$

with the concentration of the support

$$
\left\|\zeta_{0}^{s}(t, .)-\zeta_{1}^{s}(t, .)\right\|_{L^{2}\left(\left[0, x_{2}\right]\right)} \leq C e^{-\eta t} .
$$

We postpone the rather technical proof of this theorem to Appendix $\mathrm{C}$ and now turn to the numerical comparison of the full and reduced model solutions.

4. The numerical scheme. In this part, we first describe the finite volume scheme designed to simulate the PDEs of the maturity distribution in age $\zeta(t, x)(3.1)$ and mass distribution in age $\tilde{\rho}(t, x)(3.3)$, then we perform a numerical error analysis in a simplified case where we can compute the exact solution of system $(3.1,3.3)$.

4.1. Finite volume discretization. Fixing the length of the cell cycle to 1 , we denote by $\Delta_{x}$ the space step and $N_{x}$ the number of meshes. We set $\Delta_{x}=\frac{1}{N_{x}}$ and we introduce the notation for the grid points and cell centers

$$
x_{k}=k \Delta_{x}, \quad x_{k+\frac{1}{2}}=\left(k+\frac{1}{2}\right) \Delta_{x}, \text { for } k=0, \ldots, N_{x}-1 .
$$

The time discretization is defined by

$$
t^{0}=0, \quad t^{n+1}=t^{n}+\Delta_{t}, \text { for } n=0, \ldots, N_{t}-1,
$$

with $N_{t}$ such that $t^{N_{t}}=t_{\max }$. The unknowns are the approximate mean values of the maturity and mass distributions in each cell $k=0, \ldots, N_{x}-1$

$$
\zeta_{k}^{n} \approx \frac{1}{\Delta_{x}} \int_{x_{k}}^{x_{k+1}} \zeta\left(x, t^{n}\right) \mathrm{d} x, \quad \rho_{k}^{n} \approx \frac{1}{\Delta_{x}} \int_{x_{k}}^{x_{k+1}} \rho\left(x, t^{n}\right) \mathrm{d} x .
$$


Piecewise constant approximation of the nonlocal control. We define the approximation of the moments and control terms at each time step $n=0, \ldots, N_{t}$

$$
\begin{gathered}
M_{f}^{n}=\Delta_{x} \sum_{k=1}^{N_{x}} \zeta_{k}^{n} \rho_{k}^{n}, \quad M_{T}^{n}=\sum_{f=1}^{N_{f}} M_{f}^{n}, \\
U^{n}=U_{\min }+\frac{U_{\max }-U_{\min }}{1+e^{c\left(M_{T}^{n}-m\right)}}, \quad u_{f}^{n}=\left(b_{1}+\frac{1-b_{1}}{e^{b_{2}\left(b_{3}-M_{f}^{n}\right)}}\right) U^{n} .
\end{gathered}
$$

Let us remark that in the framework of the time explicit schemes that we design, these quantities are computed at each time step and considered constant during the time step, as long as the discrete unknowns have not been updated.

Initial conditions. For the initial conditions, we take the functions

$$
\zeta_{0}(x)=\zeta_{0}, \quad \rho_{0}(x)= \begin{cases}\frac{\mu_{2}}{\mu_{1}} e^{-c_{G 1} x}, & \text { if } x<x_{1}, \\ \frac{\mu_{2}}{\mu_{1}} e^{-c_{S M} x}, & \text { if } x_{1}<x,\end{cases}
$$

where $0<\zeta_{0}<y_{s}$ and

$$
\begin{gathered}
c_{G 1}=\ln \left(2\left(g_{1} U_{i n i}+g_{2}\right)\right), \quad c_{S M}=\ln \left(\frac{2}{g_{1} U_{i n i}+g_{2}}\right), \\
U_{i n i}=b_{1}+\frac{\left(1-b_{1}\right)}{1+e^{b_{2}\left(b_{3}-\zeta_{0}(x)\right)}} U_{m a x}, \quad \mu_{1}=\frac{1-e^{-\frac{c_{G 1}}{2}}}{2}+\frac{e^{-\frac{c_{S M}}{2}}-e^{-c_{S M}}}{c_{S M}},
\end{gathered}
$$

and the default value of $\mu_{2}$ is chosen so that the initial cell number is normalized

$$
\int_{0}^{x_{2}} \rho_{0}(x) \mathrm{d} x=1
$$

and

$$
\left(g_{1} U_{i n i}+g_{2}\right) \rho_{0}(0)=2 \rho_{0}\left(x_{2}\right) .
$$

The discrete values of the solution at $t=0$ are initialized using a midpoint formula, accurate at the order 2 in space

$$
\zeta_{k}^{0}=\zeta_{0}\left(x_{k+\frac{1}{2}}\right), \quad \rho_{k}^{0}=\rho_{0}\left(x_{k+\frac{1}{2}}\right) .
$$

Evolution scheme. Integration over $\left[t^{n}, t^{n+1}\right] \times\left[x_{k}, x_{k+1}\right]$ yields the first order numerical scheme. We obtain the following recursion on the approximate maturity and mass

$$
\begin{aligned}
& \zeta_{k}^{n+1}=\zeta_{k}^{n}-g\left(t^{n}, x_{k}\right) \frac{\Delta_{t}}{\Delta_{x}}\left(\zeta_{k}^{n}-\zeta_{k-1}^{n}\right)+\Delta_{t} h\left(t^{n}, x_{k}, \zeta_{k}^{n}\right), \\
& \rho_{k}^{n+1}=\rho_{k}^{n}-\frac{\Delta_{t}}{\Delta_{x}}\left[g\left(t^{n}, x_{k}\right) \rho_{k}^{n}-g\left(t^{n}, x_{k-1}\right) \rho_{k-1}^{n}\right]-\Delta_{t} \Lambda\left(t^{n}, x_{k}, \zeta_{k}^{n}\right) \rho_{k}^{n} .
\end{aligned}
$$


The periodic or doubling conditions are discretized on unknowns on meshes 0 and $N_{x}-1$ to compute $\zeta_{0}^{n+1}$ and $\rho_{0}^{n+1}$

$$
\begin{aligned}
& \zeta_{0}^{n+1}=\zeta_{0}^{n}-g\left(t^{n}, x_{1}\right) \frac{\Delta_{t}}{\Delta_{x}}\left(\zeta_{0}^{n}-\zeta_{N_{x}-1}^{n}\right)+\Delta_{t} h\left(t^{n}, x_{1}, \zeta_{0}^{n}\right), \\
& \rho_{0}^{n+1}=\rho_{0}^{n}-\frac{\Delta_{t}}{\Delta_{x}}\left[g\left(t^{n}, x_{1}\right) \rho_{0}^{n}-D g\left(t^{n}, x_{N_{x}-1}\right) \rho_{N_{x}-1}^{n}\right]-\Delta_{t} \Lambda\left(t^{n}, x_{1}, \zeta_{0}^{n}\right) \rho_{0}^{n} .
\end{aligned}
$$

with $D=2$ when $\zeta_{0}^{n+1}<y_{s}$, in order to satisfy the doubling (mitosis) condition, and $D=1$ when $\zeta_{0}^{n+1} \geq y_{s}$. The CFL condition

$$
\Delta_{t} \leq \min \left\{C F L \frac{\Delta_{x}}{\max _{x \in\left[0, x_{2}\right], t}|g(t, x)|}, \frac{1}{\max _{x \in\left[0, x_{2}\right], y \in\left[0, y_{\max }\right], t}|\Lambda(t, x, y)|}\right\},
$$

with $C F L<1$, guarantees the stability. Since $\max _{x \in\left[0, x_{2}\right], t}|g(t, x)|=1$ and $\max _{x \in\left[0, x_{2}\right], y \in\left[0, y_{\max }\right], t}|\Lambda(t, x, y)|<1$, we get

$$
\Delta_{t}=C F L \Delta_{x}
$$

4.2. Numerical error analysis of the scheme. We will show in this part that it is possible to compute the exact solution when the controls are constant: $U(t)=u_{f}(t)=U_{\max }, \forall t$ and there is no cell death. Disposing of this solution will then enable us to validate and perform an error analysis of the numerical scheme.

First, we consider the ODE

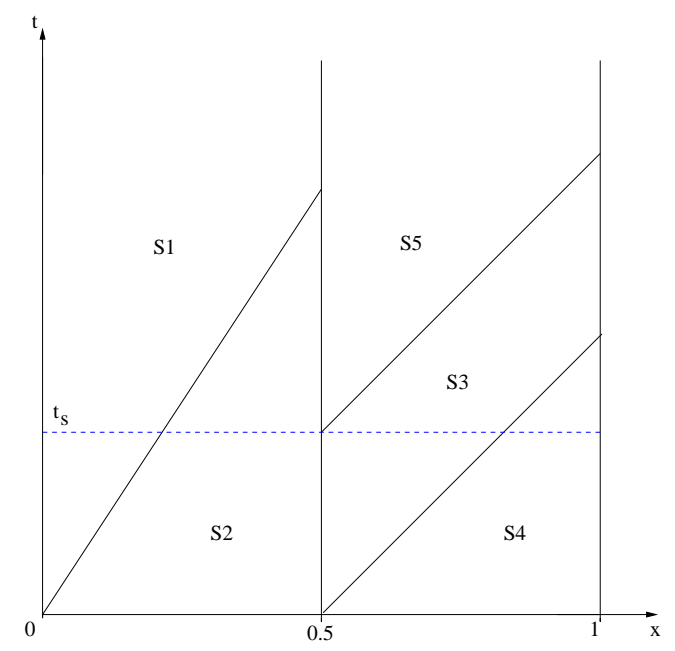

FIG. 4.1. The domain subdivisions in plane $(x, t)$ used in the definition of the exact solution $(4.4,4.5)$ in the constant control case.

$$
\left\{\begin{array}{l}
\frac{d}{d s} \bar{y}(s)=\tau_{h}\left[-\bar{y}^{2}(s)+\left(c_{1} \bar{y}(s)+c_{2}\right) \theta\left(U_{\max }\right)\right], \\
\bar{y}\left(s_{0}\right)=\zeta_{0},
\end{array}\right.
$$


where $\theta(u)=1-e^{-u / \bar{u}}$. We have that

$$
\frac{d \bar{y}}{-\bar{y}^{2}+\left(c_{1} \bar{y}+c_{2}\right) \theta\left(U_{\max }\right)}=\tau_{h} d s,
$$

which is equivalent to

$$
\left(\frac{1}{\bar{y}-k_{1}}-\frac{1}{\bar{y}-k_{2}}\right) d \bar{y}=\tau_{h}\left(k_{2}-k_{1}\right) d s,
$$

where $k_{1}, k_{2}$ are two distinct zeros of the equation $-\bar{y}^{2}+\left(c_{1} \bar{y}+c_{2}\right) \theta\left(U_{\max }\right)=0$. For any $s \geq s_{0}$, integrating both sides over $\left[s_{0}, s\right]$, we get

$$
\ln \frac{\left(\bar{y}(s)-k_{2}\right)\left(\zeta_{0}-k_{1}\right)}{\left(\bar{y}(s)-k_{1}\right)\left(\zeta_{0}-k_{2}\right)}=\tau_{h}\left(k_{2}-k_{1}\right)\left(s-s_{0}\right),
$$

which yields

$$
\bar{y}(s)=\frac{k_{2}\left(\zeta_{0}-k_{1}\right) e^{\tau_{h}\left(k_{2}-k_{1}\right)\left(s-s_{0}\right)}-k_{1}\left(\zeta_{0}-k_{2}\right)}{\left(\zeta_{0}-k_{1}\right) e^{\tau_{h}\left(k_{2}-k_{1}\right)\left(s-s_{0}\right)}-\left(\zeta_{0}-k_{2}\right)} .
$$

Considering the case $s_{0}=0$ and denoting $t_{s}$ the time such that $\bar{y}\left(t_{s}\right)=y_{s}$, we have that

$$
t_{s}=\frac{\ln \frac{\left(y_{s}-k_{1}\right)\left(\zeta_{0}-k_{2}\right)}{\left(y_{s}-k_{2}\right)\left(\zeta_{0}-k_{1}\right)}}{\tau_{h}\left(k_{2}-k_{1}\right)} .
$$

Denoting $g=g_{1} U_{\max }+g_{2}$, we find the exact solution $\zeta(t, x)$ with respect to the following subdomains, as displayed in Figure 4.1.

$$
\begin{aligned}
& S_{1}=\left\{(t, x): 0<x<x_{1}, x / g<t\right\}, \\
& S_{2}=\left\{(t, x): g t<x<x_{1}\right\}, \\
& S_{3}=\left\{(t, x): x_{1}<x<x_{2}, \quad x-x_{1}<t<x-x_{1}+t_{s}\right\}, \\
& S_{4}=\left\{(t, x): x_{1}<x<x_{2}, \quad t<x-x_{1}\right\}, \\
& S_{5}=\left\{(t, x): x_{1}<x<x_{2}, \quad x-x_{1}+t_{s}<t\right\} .
\end{aligned}
$$

It is worth noting that in $S_{3}$ and $S_{4}$ the maturity equation is a mere transport equation and the solution is constant along the characteristics $x-t=$ const. In the other subdomains, the solution is computed from the solution of the ODE (4.3). For instance, in subdomain $S_{1}$, if $\zeta(t, x)$ is solution of the $1 \mathrm{D} \operatorname{PDE}$ then $\bar{\zeta}(x ; \alpha)=\zeta\left(\frac{x}{g}+\alpha, x\right)$ is solution of equation (4.3) with the initial condition $\bar{\zeta}(0 ; \alpha)=\zeta(\alpha, 0)=\zeta\left(\alpha, x_{2}\right)$, thanks to the periodic boundary condition. Hence the solution is $\zeta(t, x)=\bar{\zeta}\left(\frac{x}{g}\right)$ with $\bar{\zeta}(t)$ solution of the ODE with initial condition $\bar{\zeta}(0)=\zeta\left(t-\frac{x}{g}, x_{2}\right)$. Using a similar reasoning for the other subdomains we obtain the exact solution $\zeta(t, x)$

\begin{tabular}{|l|l|}
\hline$(t, x)$ & $\zeta(t, x)$ \\
\hline$S_{1}$ & $\bar{y}\left(\frac{x}{g}\right)$ with initial data $\bar{y}(0)=\zeta\left(t-\frac{x}{g}, x_{2}\right)$ \\
$S_{2}$ & $\bar{y}(t)$ with initial data $\bar{y}(0)=x$ \\
$S_{3}$ & $\bar{y}\left(t-x+x_{1}\right)$ with initial data $\bar{y}(0)=x$ \\
$S_{4}$ & $x$ \\
$S_{5}$ & $\bar{y}(x)$ with initial data $\bar{y}\left(x_{1}\right)=\zeta\left(t-x+x_{1}, x_{1}\right)$ \\
\hline
\end{tabular}


Similarly, the exact solution $\tilde{\rho}(t, x)$ is given by the following formulas

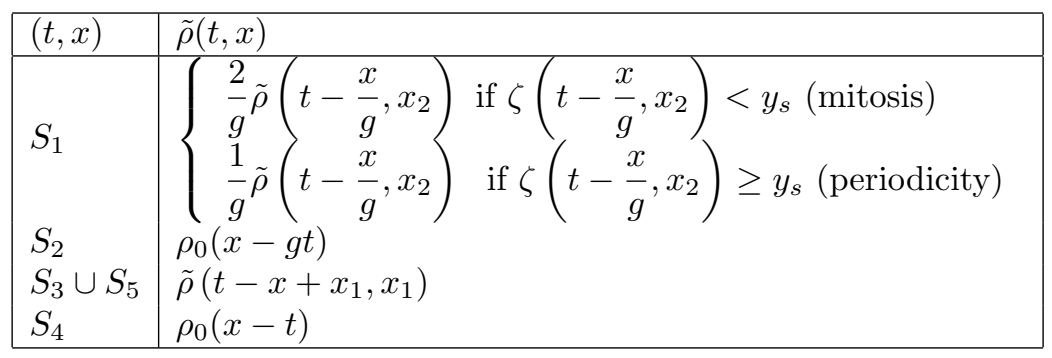

We turn to the validation of the code, which consists in verifying numerically the asymptotic order of convergence when the time step $\Delta_{t}$ and space discretization step $\Delta_{x}$ tend to zero. We compute the solution for six different space discretizations,

$$
N_{x}=200,400,800,1600,3200,6400,
$$

together with the time discretization provided by the CFL condition (4.2).

The relative errors in $L_{1}$-norm of the numerical solutions are computed using

$$
E_{\zeta}\left(\Delta_{x}, t^{n}\right)=\frac{\sum_{k=1}^{N_{x}}\left|\zeta\left(t^{n}, x_{k}\right)-\zeta_{k}^{n}\right|}{\sum_{k=1}^{N_{x}}\left|\zeta\left(t^{n}, x_{k}\right)\right|} \quad E_{\rho}\left(\Delta_{x}, t^{n}\right)=\frac{\sum_{k=1}^{N_{x}}\left|\tilde{\rho}\left(t^{n}, x_{k}\right)-\tilde{\rho}_{k}^{n}\right|}{\sum_{k=1}^{N_{x}}\left|\tilde{\rho}\left(t^{n}, x_{k}\right)\right|}
$$

The convergence behavior is illustrated in Figure 4.2 for two different times $t=0.3$
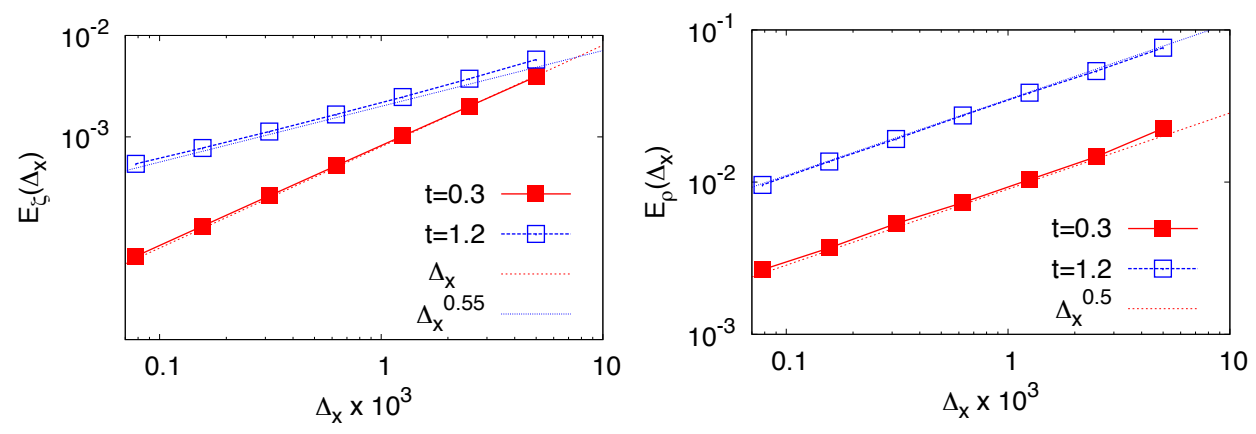

FIG. 4.2. Relative error in $L_{1}$-norm between the exact and numerical $\zeta$ (left) and $\rho$ (right) solutions at $t=0.3$ and $t=1.2$.

and $t=1.2$. At $t=0.3, \zeta$ has not yet crossed the threshold $y_{s}$. We can see that the relative error in $L_{1}$-norm for $\zeta(t, x)$ is roughly in $O\left(\Delta_{x}\right)$ while it is in $O\left(\Delta x^{0.55}\right)$ for $\rho(t, x)$. At $t=1.2, \zeta$ has already crossed the threshold $y_{s}$. The relative error in $L_{1}$-norm is in $O\left(\Delta x^{0.55}\right)$ for both components $\zeta$ and $\rho$. These numerical rates of convergence are expected for a first order finite volume scheme applied to a transport PDE with a discontinuous component. The discontinuity in $\rho\left(t, x_{1}\right)$ degrades the order of convergence from 1 to $1 / 2$ at all times for the $\rho$ component, and progressively deteriorates the order of convergence on $\zeta$, although this component is continuous.

Remark: an alternative numerical scheme derived from the conservative formulation of the 1D model in terms of $\mu(t, x)=\widetilde{\rho}(t, x) \zeta(t, x)$ has also been tested. The error study, presented in Appendix E, leads to similar results. 
We can get a rough estimate of the computational costs involved in the simulation of the reduced and 2D models by comparing a simple first order discretization in both cases, and counting the number of operations required in the inner recursion over space variables at a single time step. The original 2D model requires $\mathcal{O}\left(N_{x}^{2}\right)$ elementary operations while the reduced model requires $\mathcal{O}\left(N_{x}\right)$ elementary operations. Therefore, since $N_{x}$ is large, the $1 \mathrm{D}$ model is much faster to simulate than the $2 \mathrm{D}$ one.

5. Numerical comparison of the reduced $1 \mathrm{D}$ model with the $2 \mathrm{D}$ model. We now address the crucial point of comparing the numerical outputs of the full and reduced models. We will compare the solution of the $2 \mathrm{D}$ model computed with the initial condition

$$
\Phi_{0}(x, y)=\frac{1}{y_{\max }^{0}-y_{\min }^{0}} \rho_{0}(x) \mathbf{1}_{\left[y_{\min }^{0}, y_{\max }^{0}\right]}(x),
$$

with the solution $(\tilde{\rho}, \zeta)$ of the reduced model computed with an initial condition with the same initial age density $\tilde{\rho}(t=0, x)=\rho_{0}(x)$ and a uniform initial maturity

$$
\zeta(t=0, x)=\zeta_{0},
$$

related in some sense to $y_{\min }^{0}$ and $y_{\max }^{0}$.

We introduce some moments for the solution of the 1D model

$$
\left\{\begin{array}{l}
\bar{\rho}(t)=\int_{0}^{x_{2}} \widetilde{\rho}(t, x) \mathrm{d} x \\
\bar{M}(t)=\int_{0}^{x_{2}} \widetilde{\rho}(t, x) \zeta(t, x) \mathrm{d} x \\
\bar{\zeta}(t)=\frac{\bar{M}(t)}{\bar{\rho}(t)}
\end{array}\right.
$$

which we will compare to the moments $\rho(t), M(t)$ of the 2D solution defined by (2.33). We know from Theorem 3.1 that any solution $(\zeta, \tilde{\rho})$ defines a particular solution of the $2 \mathrm{D}$ model using the mono kinetic ansatz (2.22). Furthermore, Theorem 3.3 ensures that for long time, the support of the $2 \mathrm{D}$ solution should remain bounded in the $y$ direction by the solutions $\zeta^{i}(t, x)$, corresponding to some $s=\zeta_{i}^{0}, i=0,1$ which can be deduced from the values of $t_{s}$ and $\zeta_{i}^{s}$ for $i=0,1$ defined in the proof of Theorem $3.3^{4}$.

In this section we illustrate that in practice the comparison between the $2 \mathrm{D}$ and $1 \mathrm{D}$ model is quite robust quantitatively and satisfies the following properties

- Supp $\Phi(t, . ..) \subset\left\{(x, y), x \in\left[0, x_{2}\right], y \in\left[\zeta^{0}(t, x), \zeta^{1}(t, x)\right]\right\}$ for all times $t$.

- The average cell maturity of the 2D solution $M(t) / \rho(t)$, remains bounded by the same quantities $\bar{\zeta}^{i}(t), i=0,1$ derived from the $1 \mathrm{D}$ solutions with $\zeta_{0}^{0}=y_{\min }^{0}$ and $\zeta_{0}^{1}=y_{\max }^{0}$.

- For $y_{\max }^{0}-y_{\min }^{0}$ small enough, $(\bar{M}(t), \bar{\rho}(t))$, with $\zeta_{0}=\left(y_{\max }^{0}+y_{\min }^{0}\right) / 2$ is a good estimate of $(M(t), \rho(t))$.

- For $y_{\max }^{0}-y_{\min }^{0}$ large, $(M(t), \rho(t))$ can be estimated by a superposition of $1 \mathrm{D}$ solutions.

\footnotetext{
${ }^{4}$ Exact values for $\zeta_{i}^{0}, i=0,1$ are not available in the general case, but we will show that setting $\zeta_{0}^{0}=y_{\min }^{0}$ and $\zeta_{1}^{0}=y_{\max }^{0}$ leads to correct numerical results.
} 
5.1. Numerical illustration of Theorem 3.3. We compute the $2 \mathrm{D}$ solution with the parameters summarized in Table 5.1 and the $1 \mathrm{D}$ solutions $\zeta^{i}(t, x), i=0,1$, with initial conditions set to $\zeta_{0}^{0}=y_{\min }^{0}$ and $\zeta_{0}^{1}=y_{\max }^{0}$. In the left panel of Figure 5.1 we compare the solutions $\zeta_{i}(t, x), i=0,1$ with the minimal and maximal bounds of the support of the $2 \mathrm{D}$ solution

$$
\left\{\begin{array}{l}
\zeta_{\min 2 D}(t)=\min _{y}\{y, \Phi(t, x, y)>0\} \\
\zeta_{\max 2 D}(t)=\max _{y}\{y, \Phi(t, x, y)>0\}
\end{array}\right.
$$

which are numerically estimated by testing the FV values against a small threshold (here $10 \%$ of the current maximum density). Bounds (5.4) lie below and above the average cell maturity $M(t) / \rho(t)$ and they are enclosed between $\bar{\zeta}^{i}(t)$ for $i=0,1$ (the 1D solutions averaged over age defined in (5.3)). The center panel displays the first moment $M(t)$ of the 2D solution along with the corresponding quantities $\bar{M}^{i}(t)$, $i=0,1$, while the right panel displays the zeroth moment (total cell number). Note that both the global maturity $M(t)$ and cell number $\rho(t)$ are also bracketed by the 1D solutions $\bar{M}^{i}(t)$ and $\bar{\rho}^{i}(t), i=0,1$ but in the reverse order, with lower bounds $\bar{M}^{2}(t)$ and $\bar{\rho}^{2}(t)$, and upper bounds $\bar{M}^{1}(t)$ and $\left.\bar{\rho}^{1}(t)\right)$. This feature is not predicted by Theorem 3.3, which deals only with the behavior of $\bar{\zeta}^{i}(t)$ and $M(t) / \rho(t)$. Since $\zeta^{0}(0, x)<\zeta^{1}(0, x)$, the $1 \mathrm{D}$ solution $\left(\zeta^{0}, \tilde{\rho}^{0}\right)$ remains in the proliferative zone for a longer time than $\left(\zeta^{1}, \tilde{\rho}^{1}\right)$ and therefore the final cell number $\bar{\rho}^{0}\left(t_{\max }\right)$ is higher than $\bar{\rho}^{1}\left(t_{\max }\right)$. Theorem 3.3 can also be illustrated at the microscopic level. In Figure 2.3, the solutions $\zeta^{i}(t, x), i=0,1$, for times $t=4.37$ and $t=5$. are drawn in magenta and clearly surround the support of the $2 \mathrm{D}$ density. The long time behavior characterized by the narrowing of the support is illustrated in Figure 5.2 at $t=6.04$ and $t=8.12$. For $t=6.04$, the support shrinking property is still clearly reproduced numerically. For $t=8.12$ however, due to the numerical dispersion of the 2D solution, and the low order of approximation used to solve the 1D model, the agreement is not so good, although the part of the support corresponding to the maximum of the density is well captured by the reduced model.

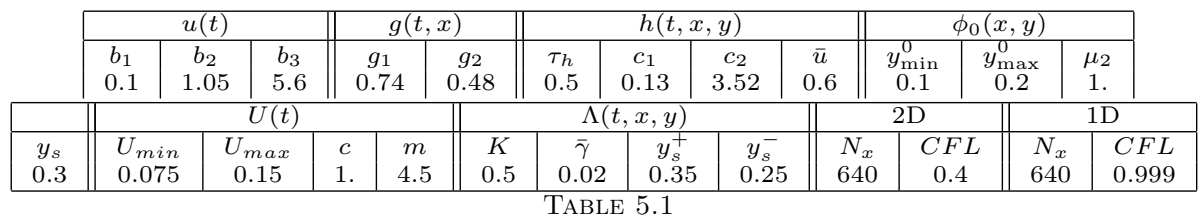

Nominal values of the parameters. The upper part corresponds to the local (follicle-specific) parameters. The lower lines gathers the global parameters in the model and the computation (discretization) parameters. The number $N_{x}$ of meshes by cell cycle in $2 D$ and $1 D$, may vary from one simulation to the other.

5.2. Approximation of the $2 \mathrm{D}$ solution moments by the $1 \mathrm{D}$ ones. Rather than obtaining lower and upper bounds it would be even more useful to directly estimate the moments of the $2 \mathrm{D}$ solution using the $1 \mathrm{D}$ model. It is quite intuitive to try with the 1D solution obtained when starting from the average initial maturity $\zeta_{0}=\frac{y_{\max }^{0}+y_{\min }^{0}}{2}$. Figure 5.3 illustrates the comparison between different macroscopic outputs for the 2D and 1D solutions, in addition to the already introduced zeroth moment (right panel) and first moment (center panel). The left panel shows the average cell maturities $\frac{M(t)}{\rho(t)}$ and $\bar{\zeta}(t)$ which are bounded below and above by 

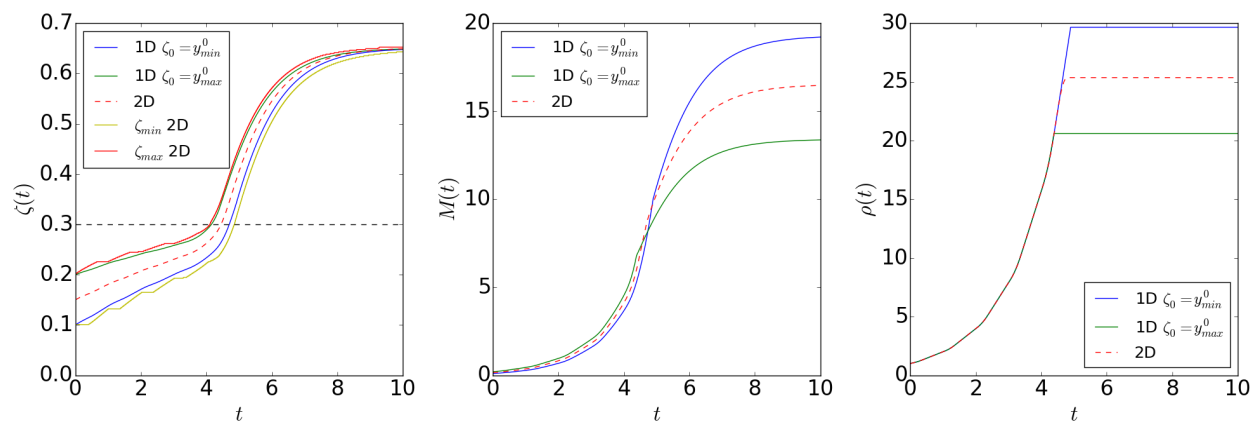

FIG. 5.1. Asymptotic behavior of $2 D$ and $1 D$ solutions in long time. The support of the initial condition of the $2 D$ model is $\left[y_{\min }^{0}, y_{\max }^{0}\right]$ in maturity. The initial condition for the $1 D$ model is $\zeta_{0}(t=0, x)=y_{\min }^{0}$ and $\zeta_{1}(t=0, x)=y_{\max }^{0}$. Left panel: $\frac{M(t)}{\rho(t)}$ (dashed red line), $\bar{\zeta}_{i}(t), i=0,1$ (blue and green solid lines), $\zeta_{\min 2 D}(t)$ (olive solid line) and $\zeta_{\max 2 D}(t)$ (red solid line). Center panel: maturity $M(t)$ (2D solution, red dashed line) and $\bar{M}_{i}(t), i=0,1$ (1D solutions, blue and green solid lines). Right panel: cell numbers $\rho(t)$ (2D solution, red dashed line) and $\bar{\rho}_{i}(t), i=0,1$ (1D solutions, blue and green solid lines).

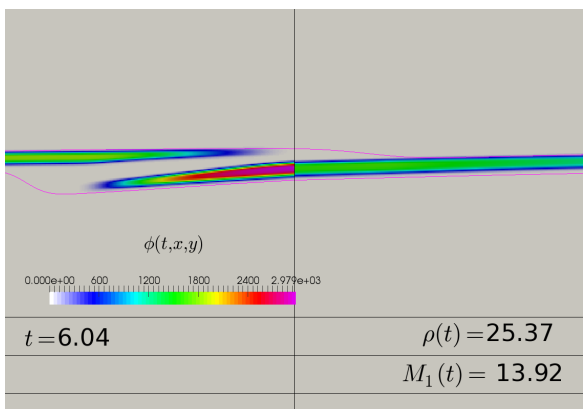

a) Snapshot at time $t=6.04$

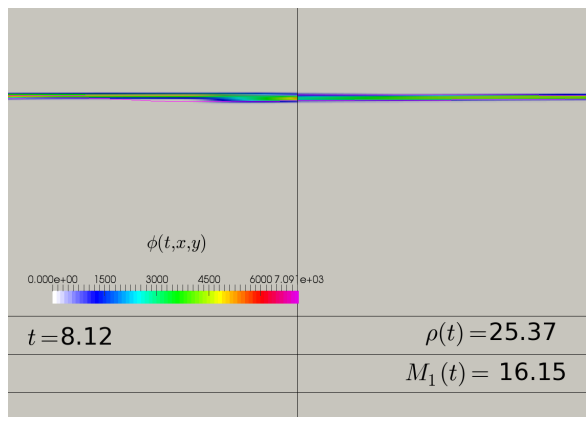

b) Snapshot at time $t=8.12$

FIG. 5.2. Snapshots of the cell density computed with the $2 D$ model at time $t=6.04$ and $t=8.12$ after exit from the cell cycle and cell death zone. Horizontal axis: age $x$ (the vertical black line separates phases $G 1$ and $S M$ at $x=0.5$ ), vertical axis: maturity $y$. The horizontal black lines indicate the maturity thresholds $y_{s}^{-}=0.25, y_{s}=0.3$ and $y_{s}^{+}=0.35$. The magenta curves correspond to the maturity solutions $\zeta(t, x)$ of the reduced model starting from $y_{\min }^{0}$ and $y_{\max }^{0}$.

$\zeta_{\min 2 D}(t)$ and $\zeta_{\max 2 D}(t)$. In each panel, the red dashed line corresponds to the 2D model solution and the blue solid line to the 1D solution.

For this intermediate thickness of the initial condition support, $y_{\min }^{0}=0.1$ and $y_{\max }^{0}=0.2$, the agreement is qualitatively quite good, but since the comparison relies on the monokinetic hypothesis, we expect that it would be best for very narrow initial conditions and deteriorates as the width of the initial condition increases.

The reduced model has been derived from the 2D solution corresponding to an initial condition with a Dirac distribution in maturity. It is therefore important to check its relevance in the general case of finite width initial condition. We denote by $M_{0.1 D}^{\text {exit }}$ (respectively $M_{02 D}^{\text {exit }}$ ) the value of the mass when all cells have a maturity higher than $\gamma_{s}^{+}$, computed with the 1D model (respectively with the 2D model). We investigate 

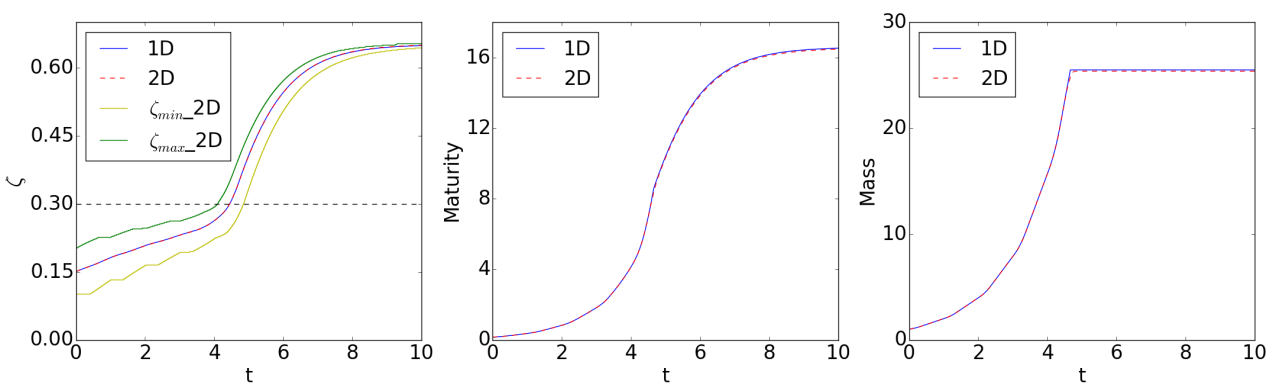

Fig. 5.3. Comparison of the $2 D$ and $1 D$ model solutions. The support of the initial condition of the $2 D$ model is $\left[y_{\min }^{0}, y_{\max }^{0}\right]$ in maturity. The initial condition for the $1 D$ model is $\zeta(t=0, x)=$ $\frac{y_{\min }^{0}+y_{\max }^{0}}{2}$. Left panel: $\frac{M(t)}{\rho(t)}$ (red dashed line), $\bar{\zeta}(t)$ (blue solid line), $\zeta_{\min 2 D}(t)$ (olive solid line) and $\zeta_{\max 2 D}(t)$ (green solid line). Center panel: maturity $M(t)$ (red dashed line) and $\bar{M}(t)$ (blue solid line). Right panel: cell numbers $\rho(t)$ (red dashed line) and $\bar{\rho}(t)$ (blue solid line).

the dependence of the absolute and relative differences

$$
E_{M}^{a}=M_{0_{-}-2 D}^{\text {exit }}-M_{0_{-} 1 D}^{\text {exit }}, \quad E_{M}^{r}=\frac{M_{0-2 D}^{\text {exit }}-M_{0_{-1}}^{\text {exit }}}{M_{0_{-2} D}^{\text {exit }}},
$$

with respect to the width and mean maturity of the $2 \mathrm{D}$ initial condition

$$
\omega_{0}=y_{\max }^{0}-y_{\min }^{0}, \quad \zeta_{0}=\frac{y_{\min }^{0}+y_{\max }^{0}}{2} .
$$

The mean maturity $\zeta_{0}$ is used as the initial condition for $\zeta$ in the corresponding $1 \mathrm{D}$ computation. First, we fix $\omega_{0}=0.1$ and let $\zeta_{0}$ vary from 0.075 to 0.225 . Such bounds are well within the range of values ensuring that the initial support does not reach $y=0$ (since $\zeta_{0}>\omega_{0} / 2=0.05$ ) and lies in the proliferation domain (since $\zeta_{0}<y_{s}-\omega_{0} / 2=0.25$ ). All other parameters remain unchanged (see Table5.1). Figure 5.4 displays the errors $E_{M}^{a}$ (blue line) and $E_{M}^{r}$ (red line) as a function of the distance $y_{s}-\zeta_{0}$ of the initial condition to the differentiation threshold. The left panel corresponds to a computation with no source term. The right panel shows the effect of cell death, with a source term coefficient $K=0.5$. The trend of these errors is actually difficult to interpret since the distance to the differentiation threshold impacts several quantities. The larger the distance is, the greater is the rate of cell proliferation, but this effect is obviously counterbalanced by others since the relative error remains within $5 \%$ in absolute value.

We then fix $\zeta_{0}=0.15$ and let $\omega_{0}$ vary from 0.02 to 0.28 . Here the maximum tested value corresponds to an initial condition covering the proliferation domain almost entirely: $y_{\min }^{0}=0.01$ and $y_{\max }^{0}=0.29$. The absolute error $E_{M}^{a}$ (blue line) and the relative error $E_{M}^{r}$ (red line) are displayed in Figure 5.5 without (left panel) or with (right panel) active source term (cell death). In this latter case the trends can be somewhat explained. The first two points corresponding to very narrow initial conditions exhibit a large error (in absolute value). This bad agreement is certainly due to the numerical inaccuracy of the Finite Volume method when the initial condition is so narrow that it can be discretized by only a few meshes. Then for small yet reasonable width, the agreement is very good even if it increases in absolute value with 


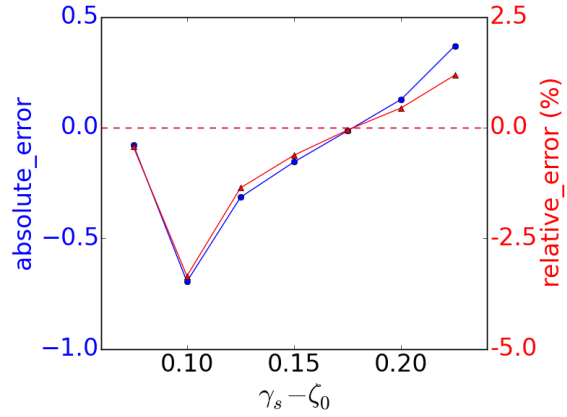

a) $K=0$. (no death)

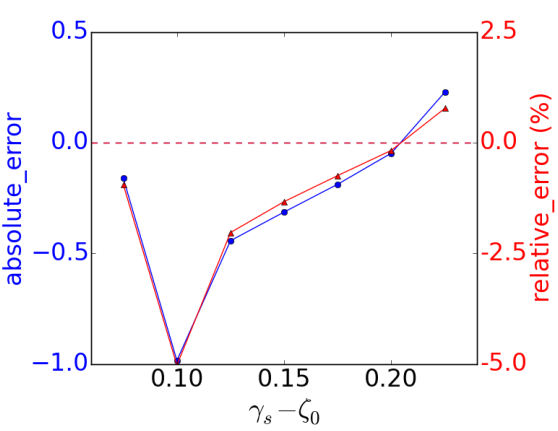

b) $K=0.5$

FIG. 5.4. The absolute (blue line) and relative (red line) signed errors between $M_{0 \_}^{\text {exit }}$ and

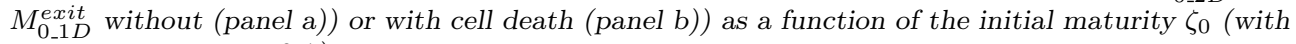
constant range $\left.\omega_{0}=0.1\right)$.

the width in maturity. The two discontinuities in that piecewise decreasing pattern come from the fact that, all things otherwise equal, when the range in maturity of the initial condition increases, the lowest maturity decreases and therefore some cells stay longer in proliferation and have to go through an additional cell cycle before reaching the differentiation threshold. In this case, as in Figure 5.4, the absolute value of the relative error remains below $5 \%$.

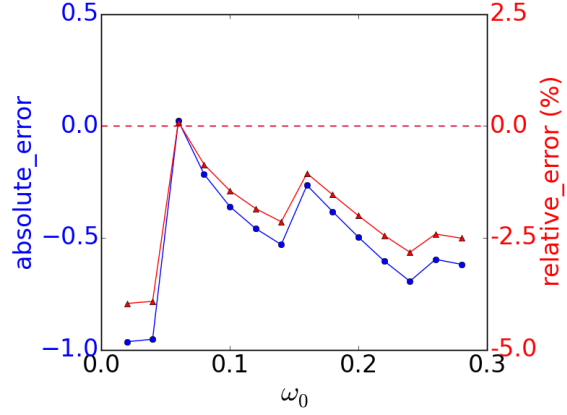

a) $K=0$. no death)

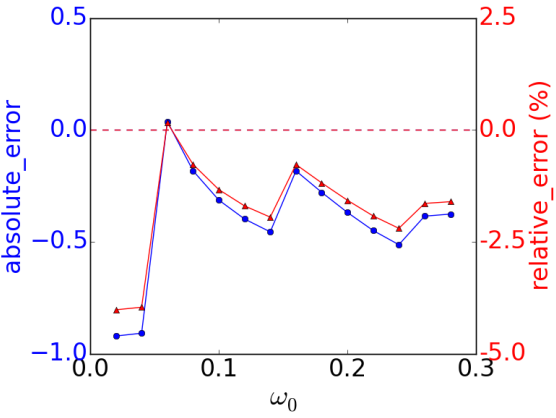

b) $K=0.5$

FIG. 5.5. The absolute (blue line) and relative (red line) signed errors between $M_{02 D}^{\text {exit }}$ and $M_{0 \_1 D}^{\text {exit }}$ without (panel a)) or with cell death (panel b)) as a function of the initial maturity range $\omega_{0}$ (with constant average initial maturity $\zeta_{0}=0.15$ ).

5.3. When the monokinetic hypothesis fails. A way to remediate. When the range in initial maturities is large, the monokinetic solution $\tilde{\Phi}(t, x, y)=$ $\widetilde{\rho}(t, x) \delta(y-\zeta(t, x))$ fails to mimic the behavior of the structured cell population. In the case when the cell death effects are not too important, we can proceed by solution superposition thanks to the quasilinear nature of the 2D PDE.

We consider, instead of the single 1D solution $(\zeta(t, x), \widetilde{\rho}(t, x))$ starting from initial condition $\zeta(t=0, x)=\left(y_{\min }^{0}+y_{\max }^{0}\right) / 2$, a "composite" $1 \mathrm{D}$ solution corresponding to the decomposition of the initial condition support into $N_{i c}$ disjoint supports of width in maturity $d=\frac{y_{\max }^{0}-y_{\min }^{0}}{N_{i c}}$. The composite solution based on $N_{i c}$ terms is defined 
as

$$
\left\{\begin{array}{l}
\bar{\rho}_{N_{i c}}(t)=\frac{1}{N_{i c}} \sum_{\ell=0}^{N_{i c}-1} \bar{\rho}^{\ell}(t) \\
\bar{M}_{N_{i c}}(t)=\frac{1}{N_{i c}} \sum_{\ell=0}^{N_{i c}-1} \bar{M}^{\ell}(t)
\end{array}\right.
$$

where $\left(\bar{\rho}^{\ell}, \bar{M}^{\ell}\right), \ell=0, \ldots, N_{i c}-1$ are the elementary solutions averaged from the 1D solutions $\left(\tilde{\rho}^{\ell}(t, x), \zeta^{\ell}(t, x)\right)$ corresponding to the initial conditions

$$
\left\{\begin{array}{l}
\widetilde{\rho}^{\ell}(0, x)=\int_{y_{\min }^{0}+\ell d}^{y_{\min }^{0}+(\ell+1) d} \Phi_{0}(x, y) d y=\frac{1}{N_{i c}} \rho_{0}(x), \\
\zeta^{\ell}(0, x)=\frac{1}{\widetilde{\rho}^{\ell}(0, x)} \int_{y_{\min }^{0}+\ell d}^{y_{\min }^{0}+(\ell+1) d} y \Phi_{0}(x, y) d y=y_{\min }^{0}+(\ell+0.5) d .
\end{array}\right.
$$

We study two effects on the superposition of 1D solutions : the non linearity in $y$ of the $h$ coefficient (2.41) and the weak non linearity due to the control $u(t)$ (2.37). In all cases we neglect the effect of the source term $\Lambda$, which we set to 0 in the simulations. We compare the results obtained with the modified $2 D$ model and by superposition of several $1 D$ solutions, in three cases : the original $h$ function (2.41), the same quadratic form for $h$ but without feed back control, which is achieved by setting

$$
h_{q}(y)=h(t, y) \text { with } u(t)=U_{\max },
$$

and finally a linear function

$$
h_{l}(t, y)=a y+b .
$$

The cell maturity level of exit from the cell cycle has been increased to $y_{s}=0.5$, to allow for a wider initial condition in the $y$ direction in the 2D case, and the parameters have been tuned so that the final cell number is about the same, around 30, in the three simulations. The parameters values departing from the ones in Table 5.1 are $g_{1}=1, \tau_{h}=0.88, y_{\min }^{0}=0.05, y_{\max }^{0}=0.45$. In the case with constant control (5.7) $\tau_{h}=0.1225$. In the $h$ linear case (5.8), $a=-0.22$ and $b=0.16$.

In Figure 5.6, we display the cell number $\rho(t)$ from the 2D solution (solid red line), along with the increasingly accurate composite solution $\bar{\rho}_{N_{i c}}(t)$, for $N_{i c}=$ $1,2,4, \ldots, 256$. The left panel corresponds to the linear case $h_{l}$ and the right panel to the quadratic case $h_{q}$. It is interesting to note that even the early transient behavior is well reproduced by the superposed solution, as soon as $N_{i c}=16$. In Figure 5.7 we display the relative error on the cell number at the end of the simulation as a function of $N_{i c}$. The relative error converges to an asymptotic value, close to $0.6 \%$ in the constant cases $h_{q}(y)$ and $h_{l}(y)$ and around $1.7 \%$ in the quadratic case with the nonlocal control $h(y, t)$. The relative error is slightly larger in this latter case due to additional nonlinear effects which prevent the superposition.

5.4. Simulation in the vector case with $N_{f}=2$. In this final example we check the behavior of our model reduction in the vector case, when $N_{f}>1$. We recall that the original biomathematical model deals with a cohort where each follicle is represented by its granulosa cell population $\Phi_{f}$. We present a simulation for $N_{f}=2$ 


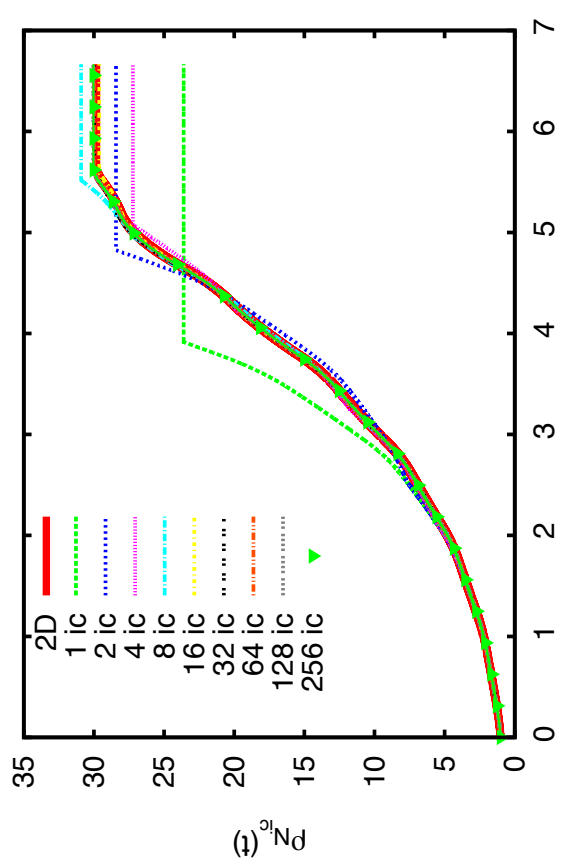

a) linear $h$

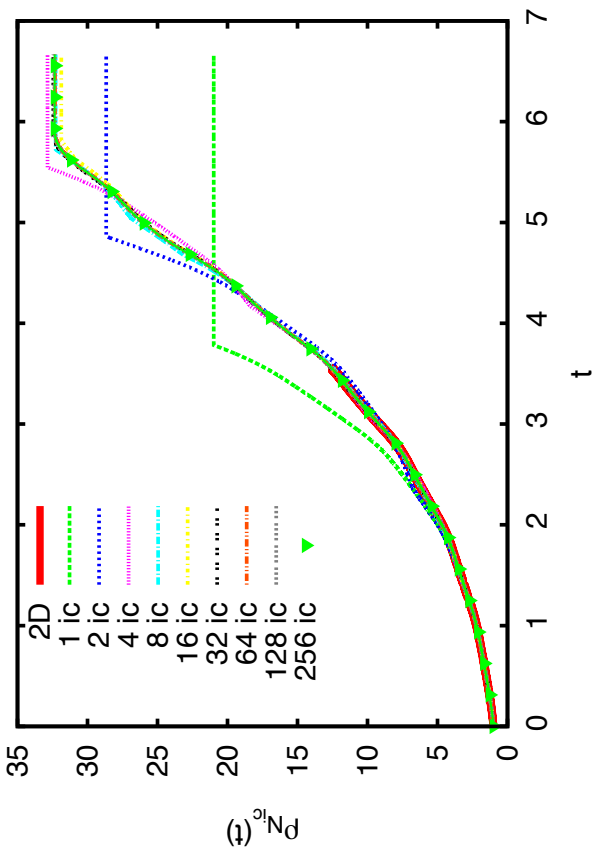

b) nonlinear $h$

FIG. 5.6. Comparison between the $2 D$ cell number $\rho(t)$ (red solid line) and $1 D$ composite solutions (5.5) $\bar{\rho}_{N_{i c}}(t)$, for $N_{i c}=1,2,4, \ldots, 256$ (thin dashed lines) in the case of constant control and no source term.
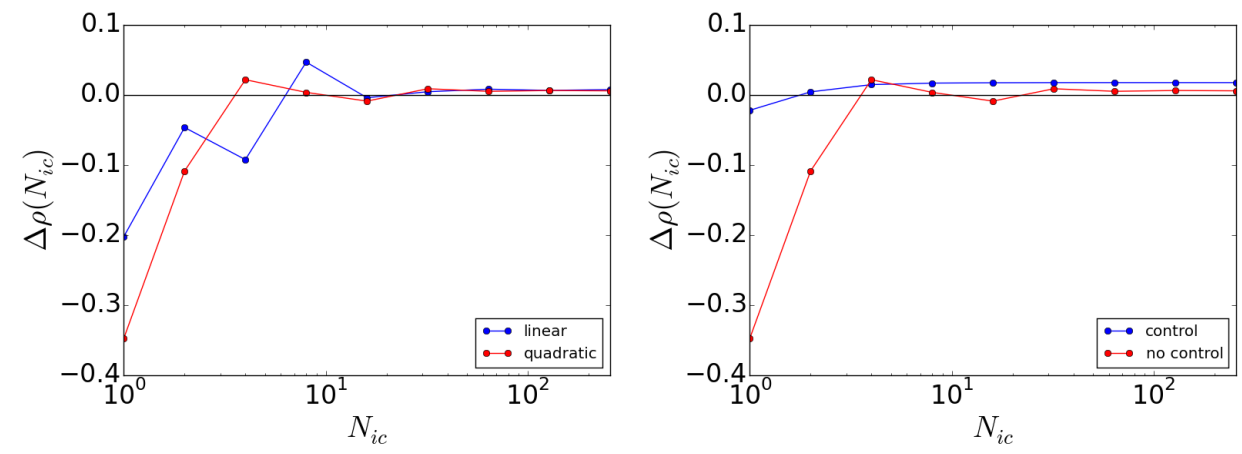

FIG. 5.7. Signed relative error between the final cell numbers in $2 D \rho(t=7)$ and composite $1 D$ solution $\bar{\rho}_{N_{i c}}(t=7)$, with respect to the number of initial conditions $N_{i c}$ (in logarithmic scale). $\Delta \rho\left(N_{i c}\right)=\frac{\tilde{\rho}_{N_{i c}}(t=7)-\rho(t=7)}{\rho(t=7)}$. Red line: original non linear $h$ with constant control; blue line, left graph: linear $h$; right graph: non linear $h$ with time varying control.

which exhibits the most interesting feature of the vector case, namely the competition between 2 follicles.

To this end, we have assigned for each follicle different values to the parameters defining the mesoscopic control $u_{f}(t)$, and the aging and maturation velocities $g_{f}(t)$, $h_{f}(t)$, as summarized in Table5.2. This choice of parameters corresponds to different ranges of values for the positive root of the maturation velocity (C.5) which plays a 
deciding role in the outcome of the simulation. At the beginning, both populations start with a normalized unit cell number, which steadily increases due to proliferation (bottom right panel). Simultaneously the cell maturity increases, since $h_{f}>0$, hence the maturity $M_{f}(t)$ increases for both follicles (upper right panel). Around $t=4$. the ovarian maturity (2.40) reaches a level around $m=21$, which triggers the drop in the global control $U(t)$ (upper left panel). The source term (2.42) then comes into play. The average cell maturity, displayed in the bottom left panel, shows that the first follicle (blue line) is from this time on right in the middle of the dangerous area $\left[y_{s}^{-}, y_{s}^{+}\right]$centered on $y_{s}=0.5$, where it remains more or less trapped, encountering massive cell death, while the second follicle (red line), rapidly crosses the dangerous zone, and stabilizes with a cell number sufficient to ensure ovulation.

We refer the reader to [3] for a thorough discussion and biological interpretation of such a simulation. What interests us here is that the complex and highly nonlinear competition phenomenon has been correctly captured by the model reduction whose numerical solution (solid lines) exhibits virtually the same features as the original 2D model solution (dashed lines).

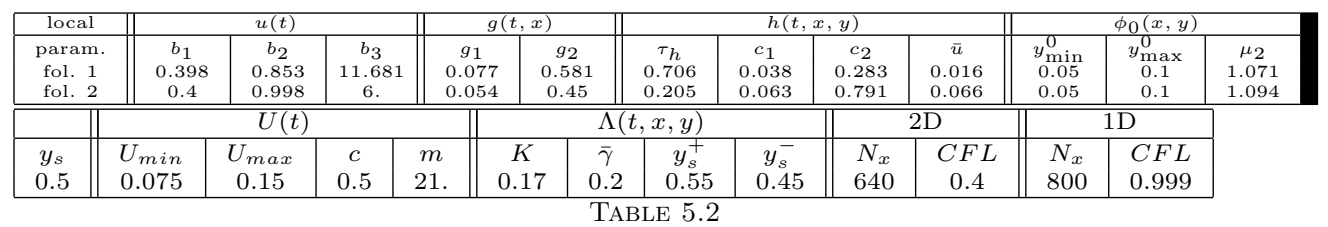

Values of the local, global and numerical parameters for the vector case simulation.
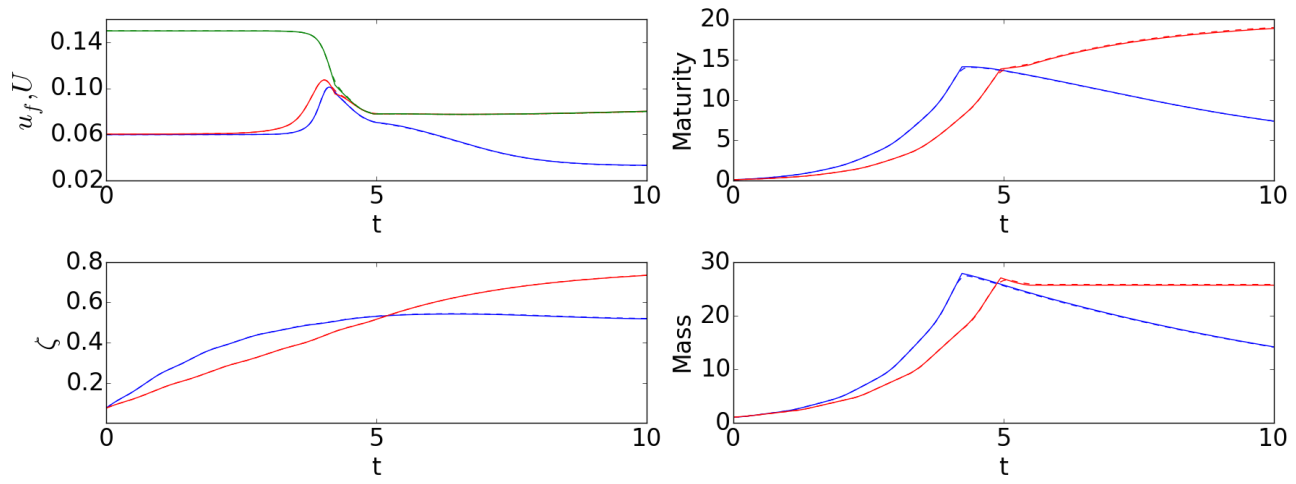

$\begin{array}{|lllll|}- & \text { follicle } 1 & \text { follicle } 2 & - & U \\ \cdots & \text { follicle } 1 \text { in 2D } & \text { follicle 2 in 2D } & - & U \text { in 2D }\end{array}$

FIG. 5.8. Macroscopic and mesoscopic outputs for a vector case, $N_{f}=2$ with the parameters of Table5.2. $f=1$ (blue), 2 (red). Top left panel: global control $U(t)$ and local controls $u_{f}(t)$ for $f=1,2$. Top right panel: maturity $M_{f}(t)$ and $\bar{M}_{f}(t)$ for $f=1,2$. Bottom left panel: average cell maturities $M_{f}(t) / \rho_{f}(t)$ and $\bar{\zeta}_{f}(t)$ for $f=1,2$. Bottom right panel: cell numbers $\rho_{f}(t)$ and $\bar{\rho}_{f}(t)$, for $f=1,2$. In each panel, the dashed lines correspond to the $2 D$ model solution and the solid lines to the $1 D$ one.

Discussion and Conclusion. We have successfully adapted the method proposed in [26] to the case of discontinuous velocity and source terms, with a cell cycle 
subdivided in subphases with distinct velocities and a localized mitosis handled by doubling the flux on a cell cycle boundary. To do so, we have introduced an intermediate 2D model and we have shown that it is equivalent in terms of moments to our original 2D model. The equivalence is exact for the zeroth order moment. For the first order moment the equivalence is exact for an affine vertical velocity $h(y)$. We have checked numerically that this latter equivalence is still valid for a quadratic velocity.

The reduced 1D model is a system of two 1D PDEs, one for the maturity $\zeta(t, x)$ and the other for the density $\widetilde{\rho}(t, x)$, which are related to the zeroth and first moments of the cell density $\Phi(t, x, y)$, solution of the original 2D system. The relations between the original and reduced models in terms of moments have been studied both theoretically and numerically, in the scalar and the vector cases. Interesting and difficult convergence results are suggested by these numerical simulations:

- The cell number and maturity $\bar{\rho}(t), \bar{M}(t)$ computed with the 1D model (in (5.3)) goes to $M_{0}(t), M_{1}(t)$ computed from the $2 \mathrm{D}$ solution for an initial condition defined in (5.1) when the width $y_{\max }^{0}-y_{\min }^{0}$ goes to zero.

- The cell number and maturity $(\bar{\rho}(t), \bar{M}(t))$, starting from the median maturity $\zeta_{0}=\left(y_{\max }^{0}+y_{\min }^{0}\right) / 2$ goes to $(\rho(t), M(t))$ when the width of the interval $y_{\max }^{0}-y_{\min }^{0}$ goes to zero.

- For a fixed initial width $y_{\max }^{0}-y_{\min }^{0}$, the composite solution $\bar{\rho}_{N_{i c}}, \bar{M}_{N_{i c}}$ defined in (5.5) goes to $\left(M_{0}(t), M_{1}(t)\right)$ when $N_{i c} \rightarrow \infty$ under specific simplifying assumptions of linearity.

Let us underline that the available theory dealing with exact solutions essentially relies on a fixed point approach [30], according to which we have no further estimate of the error between two distinct solutions coming from distinct initial data. Significant progress at the PDE level is required in order to address the error estimate issue.

We have highlighted the practical robustness of the reduction and its interest from the numerical point of view, in term of accuracy and computational speed. Due to the solution-dependent switch $\zeta(t, x)=y_{s}$ in (3.4), improving the convergence order of the numerical scheme applied to the reduced model would require a nonlinear modification of the simple first order scheme that we use here.

To investigate further the issue of parameter calibration already addressed in [3], we plan to perform intensive numerical simulations to identify various parametric configurations corresponding to specific physiological (e.g. mono-ovulation versus poly-ovulation) or pathological situations (e.g. anovulation). The sensitivity of the simulation outcomes with respect to parameter perturbations would also provide us with interesting insight on the impact and extent of heterogeneity among the follicle cohort. The $1 \mathrm{D}$ reduced model, which has a lower computing cost compared to the original 2D one, will be a welcome additional tool to embed within the numerical statistical methods that will be deployed to achieve both goals.

Acknowledgements. The authors thank Philippe Michel for helpful discussions regarding reference [26].

\section{REFERENCES}

[1] B. Aymard, F. Clément, F. Coquel, and M. Postel, Numerical simulation of the selection process of the ovarian follicles, ESAIM: Proc., 38 (2012), pp. 99-117.

[2] B. Aymard, F. Clément, F. Coquel, and M. Postel, A numerical method for transport equations with discontinuous flux functions: Application to mathematical modeling of cell dynamics, SIAM J. Sci. Comput., 35 (2013), pp. 2442-2468. 
[3] B. Aymard, F. Clément, D. Monniaux, and M. Postel, Cell-kinetics based calibration of a multiscale model: Application to cell population dynamics in ovarian follicles. submitted, hal-01186381, Aug. 2015.

[4] B. Aymard, F. Clément, and M. Postel, Adaptive mesh refinement strategy for a non conservative transport problem, ESAIM Math. Model. Numer. Anal., 90 (2014), pp. 13811412.

[5] J. Bestel, F. Clément, And M. Sorine, A biomechanical model of muscle contraction, in Medical Image Computing and Computer-Assisted Intervention-MICCAI 2001, Springer, 2001, pp. 1159-1161.

[6] F. Bouchut, On zero pressure gas dynamics, in Advances in kinetic theory and computing, vol. 22 of Ser. Adv. Math. Appl. Sci., World Sci. Publ., River Edge, NJ, 1994, pp. 171-190.

[7] F. Bouchut and F. James, One-dimensional transport equations with discontinuous coefficients, Nonlinear Analysis, TMA, 32 (1998), pp. 891-933.

[8] Y. BREnier AND L. CorRias, A kinetic formulation for multi-branch entropy solutions of scalar conservation laws, Ann. Inst. Henri Poincaré, Analyse non linéaire, 15 (1998), pp. 169-190.

[9] Y. Brenier And E. Grenier, Sticky particles and scalar conservation laws, SIAM J. Numer. Anal., 35 (1998), pp. 2317-2328 (electronic).

[10] C. Chalons, D. Kah, and M. Massot, Beyond pressureless gas dynamics: quadrature-based velocity moment models, Commun. Math. Sci., 10 (2012), pp. 1241-1272.

[11] F. Clément and D. Monniaux, Multiscale modelling of ovarian follicular selection, Progress in Biophysics and Molecular Biology, 113 (2013), pp. 398-408.

[12] F. Colombini, G. Crippa, And J. Rauch, A note on two-dimensional transport with bounded divergence, Communications in Partial Differential Equations, 31 (2006), pp. 1109-1115.

[13] R. M. Colombo and M. Lécureux-Mercier, Nonlocal crowd dynamics models for several populations, Acta Mathematica Scientia, 32 (2012), pp. 177-196.

[14] P. Degond, A. Frouvelle, And J.-G. Liu, Phase transitions, hysteresis, and hyperbolicity for self-organized alignment dynamics, Arch. Ration. Mech. Anal., 216 (2015), pp. 63-115.

[15] J Dyson, R Villella-Bressan, and G WebB, A spatial model of tumor growth with cell age, cell size, and mutation of cell phenotypes, Mathematical Modelling of Natural Phenomena, 2 (2007), pp. 69-100.

[16] N. Echenim, D. Monniaux, M. Sorine, And F. Clément, Multi-scale modeling of the follicle selection process in the ovary, Math. Biosci., 198 (2005), pp. 57-79.

[17] E. Godlewski and P-A. Raviart, Numerical Approximation of Hyperbolic Systems of Conservation Laws, vol. 118, Springer, 1996.

[18] L. Gosse And F. James, Numerical approximations of one-dimensional linear conservation equations with discontinuous coefficients, Mathematics of Computation, 69 (2000), pp. pp. $987-1015$.

[19] Laurent Gosse and Peter A. Markowich, Multiphase semiclassical approximation of an electron in a one-dimensional crystalline lattice. i. homogeneous problems, Journal of Computational Physics, 197 (2004), pp. 387-417.

[20] K. T. JosEPH, Explicit solutions for a system of first-order partial differential equations., Electronic Journal of Differential Equations (EJDE) [electronic only], 2008 (2008), pp. Paper No. 157,8 p., electronic only-Paper No. 157,8 p., electronic only.

[21] A. Klar, N. Marheineke, and R. Wegener, Hierarchy of mathematical models for production processes of technical textiles, ZAMM-Journal of Applied Mathematics and Mechanics/Zeitschrift für Angewandte Mathematik und Mechanik, 89 (2009), pp. 941-961.

[22] D. C. Levermore, Moment closure hierarchies for kinetic theories, Journal of Statistical Physics, 83 (1996), pp. 1021-1065.

[23] P.-L. Lions, B. Perthame, and E. Tadmor, A kinetic formulation of multidimensional scalar conservation laws and related equations, J. Amer. Math. Soc., 7 (1994), pp. 169-191.

[24] - Kinetic formulation of the isentropic gas dynamics and p-systems, Comm. Math. Phys., 163 (1994), pp. 415-431.

[25] D. L. MARChisio AND R. O. Fox, Solution of population balance equations using the direct quadrature method of moments, Journal of Aerosol Science, 36 (2005), pp. 43-73.

[26] P. Michel, Multiscale modeling of follicular ovulation as a mass and maturity dynamical system, Multiscale Model. Simul., 9 (2011), pp. 282-313.

[27] I. Müller and T. Ruggeri, Extended thermodynamics, vol. 37 of Springer Tracts in Natural Philosophy, Springer-Verlag, New York, 1993.

[28] B. Perthame, Kinetic formulation of conservation laws, vol. 21 of Oxford Lecture Series in Mathematics and its Applications, Oxford University Press, Oxford, 2002.

[29] B. Perthame, Transport Equations in Biology, Frontiers in Mathematics, Birkhauser Verlag, 2007. 
[30] P. Shang, Cauchy problem for multiscale conservation laws: Application to structured cell populations, J. Math. Anal. Appl., 401 (2013), pp. 896-920.

[31] P. Shang And Z. WAng, Analysis and control of a scalar conservation law modeling a highly re-entrant manufacturing system, J. Differential Equations, 2 (2011), pp. 949-982.

[32] H. Struchtrup, Failures of the burnett and super-burnett equations in steady state processes, Continuum Mechanics and Thermodynamics, 17 (2005), pp. 43-50.

[33] L. Wang, M. B. Short, And A. L. BertozzI, Efficient numerical methods for multiscale crowd dynamics with emotional contagion, Mathematical Models and Methods in Applied Sciences, 27 (2017), pp. 205-230. 


\section{Appendix A. Asymptotic convergence in time for the toy model.}

Proof. of Theorem 2.1. Conditions $(2.3,2.4)$ imply that there exists $C>0$ such that for all $t>0|h(t, y)| \leq C(1+|y|)$ which in turns ensures that the characteristic curve $Y\left(t ; y_{0}\right)$ solution of $(2.7)$ is defined for all $t<+\infty$ and all $y_{0} \in \mathbb{R}$. Indeed the solution of (2.1) can be derived

$$
\phi\left(t, Y\left(t, y_{0}\right)\right)=\phi_{0}\left(y_{0}\right) \exp \left(-\int_{0}^{t} \partial_{y} h\left(\tau, Y\left(\tau, y_{0}\right)\right) \mathrm{d} \tau\right),
$$

and, along with (2.2), it implies that for all $T>0$ there exists $0<K_{T}<\infty$ such that $\operatorname{Supp}(\phi(t,).) \subset\left[-K_{T}, K_{T}\right]$.

We first show a preliminary property of the solutions of (2.1) and (2.7)

Lemma A.1. For all $\phi(t, y)$ solution of (2.1,2.2), for all $\zeta(t)$ solution of (2.7), for all $F \in C^{1}(\mathbb{R})$ positive, we have

$$
\frac{d}{d t} \int_{\mathbb{R}} \phi(t, y) F(y-\zeta(t)) d y=\int_{\mathbb{R}} \phi(t, y) F^{\prime}(y-\zeta(t))(h(t, y)-h(t, \zeta(t))) d y .
$$

Proof.

$$
\begin{aligned}
\frac{d}{d t} \int_{\mathbb{R}} \phi(t, y) F(y-\zeta(t)) \mathrm{d} y= & \int_{\mathbb{R}} F(y-\zeta(t)) \partial_{t} \phi \mathrm{d} y-\int_{\mathbb{R}} \phi \zeta^{\prime}(t) F^{\prime}(y-\zeta(t)) \mathrm{d} y \\
= & \int_{\mathbb{R}} F(y-\zeta(t))\left(-\partial_{y}(h \phi)\right) \mathrm{d} y-\int_{\mathbb{R}} \phi \zeta^{\prime}(t) F^{\prime}(y-\zeta(t)) \mathrm{d} y \\
= & -[F(y-\zeta(t)) h(t, y) \phi(t, y)]_{\mathbb{R}}+\int_{\mathbb{R}} F^{\prime}(y-\zeta(t)) h(t, y) \phi(t, y) \mathrm{d} y \\
& -\int_{\mathbb{R}} \phi(t, y) h(t, \zeta(t)) F^{\prime}(y-\zeta(t)) \mathrm{d} y \\
= & \int_{\mathbb{R}} \phi(t, y) F^{\prime}(y-\zeta(t))(h(t, y)-h(t, \zeta(t))) \mathrm{d} y .
\end{aligned}
$$

We apply Lemma A.1 for $F(z)=\left(z_{+}\right)^{2}$ (with $\left.z_{+}=\max (z, 0)\right)$ and $\zeta=\zeta_{2}$ (the solution of $(2.7)$ for $\left.\zeta_{2}(0)=y_{\max }^{0}\right)$

$$
\frac{d}{d t} \int_{\mathbb{R}} \phi(t, y)\left(\left(y-\zeta_{2}(t)\right)_{+}\right)^{2} \mathrm{~d} y=2 \int_{\mathbb{R}}\left(\phi(t, y)\left(\left(y-\zeta_{2}(t)\right)_{+}\right)\left(h(t, y)-h\left(t, \zeta_{2}(t)\right)\right)\right) \mathrm{d} y .
$$

Using $y$ differentiability of $h$ yields

$$
\frac{d}{d t} \int_{\mathbb{R}} \phi(t, y)\left(\left(y-\zeta_{2}(t)\right)_{+}\right)^{2} \mathrm{~d} y=2 \int_{\mathbb{R}}\left(\phi(t, y)\left(\left(y-\zeta_{2}(t)\right)_{+}\right) \int_{\zeta_{2}(t)}^{y} \partial_{y} h(t, z) \mathrm{d} z\right) \mathrm{d} y,
$$

and using the fact that $\phi(t, y) \geq 0$ since $\phi_{0}(y) \geq 0$ we obtain

$$
\frac{d}{d t} \int_{\mathbb{R}} \phi(t, y)\left(\left(y-\zeta_{2}(t)\right)_{+}\right)^{2} \mathrm{~d} y \leq-2 \eta \int_{\mathbb{R}}\left(\phi(t, y)\left(\left(y-\zeta_{2}(t)\right)_{+}\right)^{2}\right) \mathrm{d} y,
$$

hence, integrating between 0 and $t$

$$
0 \leq \int_{\mathbb{R}} \phi(t, y)\left(\left(y-\zeta_{2}(t)\right)_{+}\right)^{2} \mathrm{~d} y \leq e^{-2 \eta t} \int_{\mathbb{R}}\left(\phi_{0}(y)\left(\left(y-y_{\max }^{0}\right)_{+}\right)^{2}\right) \mathrm{d} y=0,
$$


which implies that $\phi(t, y)=0$ for all $y \geq \zeta_{2}(t)$. Similarly, using $F(z)=\left(z_{-}\right)^{2}$ (with $\left.z_{-}=\min (z, 0)\right)$ and $\zeta=\zeta_{1}$, we show that $\phi(t, y)=0$ for all $y \leq \zeta_{1}(t)$, which completes the proof of (2.8). Then to obtain (2.9) we write

$$
\begin{aligned}
\frac{d}{d t}\left(\zeta_{2}(t)-\zeta_{1}(t)\right)^{2} & =2\left(\zeta_{2}(t)-\zeta_{1}(t)\right) \frac{d}{d t}\left(\zeta_{2}(t)-\zeta_{1}(t)\right) \\
& =2\left(\zeta_{2}(t)-\zeta_{1}(t)\right)\left(h\left(t, \zeta_{2}(t)\right)-h\left(t, \zeta_{1}(t)\right)\right) \\
& =2\left(\zeta_{2}(t)-\zeta_{1}(t)\right) \int_{\zeta_{1}(t)}^{\zeta_{2}(t)} \partial_{y} h(t, y) \mathrm{d} y \\
\frac{d}{d t}\left(\zeta_{2}(t)-\zeta_{1}(t)\right)^{2} & \leq-2 \eta\left(\zeta_{2}(t)-\zeta_{1}(t)\right)^{2} \\
\left(\zeta_{2}(t)-\zeta_{1}(t)\right)^{2} & \leq e^{-2 \eta t}\left(y_{\max }^{0}-y_{\min }^{0}\right)^{2} .
\end{aligned}
$$

Proof. of Theorem 2.2. We now endeavour to show that the measure $\phi^{a}$ is a weak solution of $(2.1)$ in $\mathcal{D}^{\prime}([0,+\infty[\times \mathbb{R})$, where $\mathcal{D}([0,+\infty[\times \mathbb{R})$ is the set of infinitely differentiable functions compactly supported in $t$ and $y$. We multiply (2.1) by a test function $\psi \in \mathcal{D}$ and integrate over $[0,+\infty[\times \mathbb{R}$.

$$
\int_{0}^{+\infty} \int_{\mathbb{R}} \psi\left(\partial_{t} \phi+\partial_{y}(h \phi)\right) \mathrm{d} y \mathrm{~d} t=0
$$

Integration by parts yields

$$
\int_{\mathbb{R}}[\psi \phi]_{t=0}^{t=+\infty} \mathrm{d} y+\int_{0}^{+\infty}[h \psi \phi]_{\mathbb{R}} \mathrm{d} t=\int_{0}^{+\infty} \int_{\mathbb{R}} \phi\left(\partial_{t} \psi+h \partial_{y} \psi\right) \mathrm{d} y \mathrm{~d} t
$$

which simplifies thanks to support compactness into

$$
\int_{0}^{+\infty} \int_{\mathbb{R}} \phi\left(\partial_{t} \psi+h \partial_{y} \psi\right) \mathrm{d} y \mathrm{~d} t=-\int_{\mathbb{R}} \phi_{0}(y) \psi(0, y) d y .
$$

On the other hand, any $\zeta(t)$ solution of (2.7) satisfies

$$
\begin{aligned}
\int_{0}^{+\infty} \int_{\mathbb{R}} \delta(y-\zeta(t))\left(\partial_{t} \psi(t, y)+\right. & \left.h(t, y) \partial_{y} \psi(t, y)\right) \mathrm{d} y \mathrm{~d} t= \\
& \int_{\mathbb{R}}\left(\partial_{t} \psi(t, \zeta(t))+h(t, \zeta(t)) \partial_{y} \psi(t, \zeta(t))\right) \mathrm{d} t \\
= & \int_{\mathbb{R}}\left(\partial_{t} \psi(t, \zeta(t))+\zeta^{\prime}(t) \partial_{y} \psi(t, \zeta(t))\right) \mathrm{d} t \\
= & \int_{\mathbb{R}} \frac{d}{d t} \psi(t, \zeta(t)) \mathrm{d} t \\
= & -\psi(0, \zeta(0))=-\int_{\mathbb{R}} \delta(y-\zeta(0)) \psi(0, y) \mathrm{d} y
\end{aligned}
$$

Therefore the measures $\phi^{a}(t)=M_{0} \delta(y-\zeta(t))$ satisfy (A.3) with $\phi_{0}(y)=M_{0} \delta(y-$ $\zeta(0))$.

Appendix B. Proof of weak formulation for the modified setup.

Proof. of Lemma 3.2 Each scalar PDE in the system (2.10) is well posed wherever $g(t, x)$, and $h(t, x, y)$ are continuous. We write the weak formulation in each of the 
four sub-domains $G 1, S M$ and $D_{1}$ and $D_{2}$, by multiplying the original PDE by a test function $\psi(t, x, y) \in C^{\infty}\left(\left[0,+\infty\left[\times\left[0, x_{2}\right] \times \mathbb{R}^{+}\right)\right.\right.$, compactly supported in time and $y$ and $x_{2}$-periodic in $x$. On the one hand, since $\Phi$ satisfies (2.10) we have

$$
I=\int_{0}^{+\infty} \int_{0}^{x_{2}} \int_{\mathbb{R}^{+}} \psi\left(\partial_{t} \Phi+\partial_{x}(g \Phi)+\partial_{y}(h \Phi)\right) \mathrm{d} y \mathrm{~d} x \mathrm{~d} t=-\int_{0}^{+\infty} \int_{0}^{x_{2}} \int_{\mathbb{R}^{+}} \psi \Phi \Lambda \mathrm{d} y \mathrm{~d} x \mathrm{~d} t .
$$

On the other hand we split $I$ into four terms and integrate by parts in $x$ and $y$ :

$$
\begin{aligned}
I_{G 1}= & \int_{0}^{x_{1}} \int_{0}^{y_{s}}\left(\partial_{t} \Phi+\partial_{x}(g \Phi)+\partial_{y}(h \Phi)\right) \psi \mathrm{d} y \mathrm{~d} x \\
= & \int_{0}^{x_{1}} \int_{0}^{y_{s}}\left(\partial_{t}(\Phi \psi)-g \Phi \partial_{x} \psi-h \Phi \partial_{y} \psi\right) \mathrm{d} y \mathrm{~d} x+\int_{0}^{y_{s}}[g \Phi \psi]_{0}^{x_{1}} \mathrm{~d} y+\int_{0}^{x_{1}}[h \Phi \psi]_{0}^{y_{s}} \mathrm{~d} x \\
= & \int_{0}^{x_{1}} \int_{0}^{y_{s}}\left(\partial_{t}(\Phi \psi)-g \Phi \partial_{x} \psi-h \Phi \partial_{y} \psi\right) \mathrm{d} y \mathrm{~d} x \\
& +\int_{0}^{y_{s}}\left(g\left(t, x_{1}^{-}\right) \Phi\left(t, x_{1}^{-}, y\right) \psi\left(t, x_{1}, y\right)-g\left(t, 0^{+}\right) \Phi\left(t, 0^{+}, y\right) \psi(t, 0, y)\right) \mathrm{d} y \\
& +\int_{0}^{x_{1}}\left(h\left(t, x, y_{s}^{-}\right) \Phi\left(t, x, y_{s}^{-}\right) \psi\left(t, x, y_{s}\right)-h\left(t, x, 0^{+}\right) \Phi\left(t, x, 0^{+}\right) \psi(t, x, 0)\right) \mathrm{d} x,
\end{aligned}
$$

where the last term cancels thanks to (2.18). Similarly over subdomain $S M$ we get

$$
\begin{aligned}
I_{S M}= & \int_{x_{1}}^{x_{2}} \int_{0}^{y_{s}}\left(\partial_{t}(\Phi \psi)-g \Phi \partial_{x} \psi-h \Phi \partial_{y} \psi\right) \mathrm{d} y \mathrm{~d} x \\
& +\int_{0}^{y_{s}}\left(g\left(t, x_{2}^{-}\right) \Phi\left(t, x_{2}^{-}, y\right) \psi\left(t, x_{2}, y\right)-g\left(t, x_{1}^{+}\right) \Phi\left(t, x_{1}^{+}, y\right) \psi\left(t, x_{1}, y\right)\right) \mathrm{d} y \\
& +\int_{x_{1}}^{x_{2}}\left(h\left(t, x, y_{s}^{-}\right) \Phi\left(t, x, y_{s}\right) \psi\left(t, x, y_{s}\right)-h\left(t, x, 0^{+}\right) \Phi\left(t, x, 0^{+}\right) \psi(t, x, 0)\right) \mathrm{d} x .
\end{aligned}
$$

Then we integrate over subdomains $D_{1}$ and $D_{2}$

$$
\begin{aligned}
& I_{D_{1}}=\int_{0}^{x_{1}} \int_{y_{s}}^{+\infty}\left(\partial_{t}(\Phi \psi)-g \Phi \partial_{x} \psi-h \Phi \partial_{y} \psi\right) \mathrm{d} y \mathrm{~d} x \\
& +\int_{y_{s}}^{+\infty}\left(g\left(t, x_{1}^{-}\right) \Phi\left(t, x_{1}^{-}, y\right) \psi\left(t, x_{1}, y\right)-g\left(t, 0^{+}\right) \Phi\left(t, 0^{+}, y\right) \psi(t, 0, y)\right) \mathrm{d} y \\
& +\int_{0}^{x_{1}}\left(h(t, x,+\infty) \Phi(t, x,+\infty) \psi(t, x,+\infty)-h\left(t, x, y_{s}^{+}\right) \Phi\left(t, x, y_{s}^{+}\right) \psi\left(t, x, y_{s}\right)\right) \mathrm{d} x
\end{aligned}
$$

and

$$
\begin{aligned}
& I_{D_{2}}=\int_{x_{1}}^{x_{2}} \int_{y_{s}}^{+\infty}\left(\partial_{t}(\Phi \psi)-g \Phi \partial_{x} \psi-h \Phi \partial_{y} \psi\right) \mathrm{d} y \mathrm{~d} x \\
& +\int_{y_{s}}^{+\infty}\left(g\left(t, x_{2}^{-}\right) \Phi\left(t, x_{2}^{-}, y\right) \psi\left(t, x_{2}, y\right)-g\left(t, x_{1}^{+}\right) \Phi\left(t, x_{1}^{+}, y\right) \psi(t, 0, y)\right) \mathrm{d} y \\
& +\int_{x_{1}}^{x_{2}}\left(h(t, x,+\infty) \Phi(t, x,+\infty) \psi(t, x,+\infty)-\underline{\left.h\left(t, x, y_{s}^{+}\right) \Phi\left(t, x, y_{s}^{+}\right) \psi\left(t, x, y_{s}\right)\right)} \mathrm{d} x .\right.
\end{aligned}
$$

We sum $I_{G 1}, I_{S M}, I_{D_{1}}$ and $I_{D_{2}}$. We reorganize the boundary terms and use the 
test function periodicity in $x$

$$
\begin{aligned}
I= & \int_{0}^{x_{2}} \int_{0}^{+\infty}\left(\partial_{t}(\Phi \psi)-g \Phi \partial_{x} \psi-h \Phi \partial_{y} \psi\right) \mathrm{d} y \mathrm{~d} x \\
& +\int_{0}^{y_{s}}\left(g\left(t, x_{1}^{-}\right) \Phi\left(t, x_{1}^{-}, y\right)-g\left(t, x_{1}^{+}\right) \Phi\left(t, x_{1}^{+}, y\right)\right) \psi\left(t, x_{1}, y\right) \\
& +\int_{0}^{y_{s}}\left(g\left(t, x_{2}^{-}\right) \Phi\left(t, x_{2}^{-}, y\right)-g\left(t, 0^{+}\right) \Phi\left(t, 0^{+}, y\right)\right) \psi(t, 0, y) \mathrm{d} y \\
& +\int_{0}^{x_{1}}\left(h\left(t, x, y_{s}^{-}\right) \Phi\left(t, x, y_{s}^{-}\right)-h\left(t, x, y_{s}^{+}\right) \Phi\left(t, x, y_{s}^{+}\right)\right) \psi\left(t, x, y_{s}\right) \mathrm{d} x \\
& +\int_{y_{s}}^{+\infty}\left(g\left(t, x_{2}^{-}\right) \Phi\left(t, x_{2}^{-}, y\right)-g\left(t, 0^{+}\right) \Phi\left(t, 0^{+}, y\right)\right) \psi(t, 0, y) \mathrm{d} y \\
& +\int_{y_{s}}^{+\infty}\left(g\left(t, x_{1}^{-}\right) \Phi\left(t, x_{1}^{-}, y\right)-g\left(t, x_{1}^{+}\right) \Phi\left(t, x_{1}^{+}, y\right)\right) \psi\left(t, x_{1}, y\right) \mathrm{d} y .
\end{aligned}
$$

The first boundary integral (B.2) cancels thanks to (2.12), the third one (B.4) cancels thanks to the continuity of $h$ over $G 1-D$, the fourth one (B.5) cancels thanks to the x-periodicity of $g \Phi(2.31)$ and the last one (B.6) cancels thanks to the x-continuity of $g \Phi$ between $D_{1}$ and $D_{2}(2.32)$. Using (2.13) we obtain finally

$$
\begin{aligned}
I & =\int_{0}^{x_{2}} \int_{0}^{+\infty}\left(\partial_{t}(\Phi \psi)-g \Phi \partial_{x} \psi-h \Phi \partial_{y} \psi\right) \mathrm{d} y \mathrm{~d} x-\frac{1}{2} \int_{0}^{y_{s}} g\left(0^{+}\right) \Phi\left(t, 0^{+}, y\right) \psi(t, 0, y) \mathrm{d} y \\
& =-\int_{0}^{x_{2}} \int_{0}^{+\infty} \Lambda \Phi \psi \mathrm{d} x \mathrm{~d} y .
\end{aligned}
$$

By integrating in time we obtain

$$
\begin{aligned}
& \int_{0}^{+\infty} \int_{0}^{x_{2}} \int_{0}^{+\infty}\left(\partial_{t}(\Phi \psi)-\Phi\left(g \partial_{x} \psi+h \partial_{y} \psi\right)\right) \mathrm{d} y \mathrm{~d} x \mathrm{~d} t \\
& -\frac{1}{2} \int_{0}^{+\infty} \int_{0}^{y_{s}} g\left(t, 0^{+}\right) \Phi\left(t, 0^{+}, y\right) \psi(t, 0, y) \mathrm{d} y \mathrm{~d} t=-\int_{0}^{+\infty} \int_{0}^{x_{2}} \int_{0}^{+\infty} \Lambda \Phi \psi \mathrm{d} y \mathrm{~d} x \mathrm{~d} t,
\end{aligned}
$$

which yields (3.5) after integrating by parts in time the first product $\partial_{t} \Phi \psi$.

口

Appendix C. Proof of convergence in maturity. The proof of Theorem 3.3 follows that of [26] and we will endeavour to highlight the points where our hypotheses differ from there.

First of all, we state some properties derived from our specific choice for the control function and maturation velocities.

Since the global control $U(t)$ is bounded by $U_{\min } \leq U(t) \leq U_{\max }, \quad \forall t$, the local control

$$
u(t)=\left(b_{1}+\frac{1-b_{1}}{1+e^{b_{2}\left(b_{3}-M(t)\right)}}\right) U(t),
$$

is also bounded and we have that

$$
u_{\min }:=b_{1} U_{\min } \leq u(t) \leq u_{\max }:=U_{\max }, \quad \forall t .
$$


For our specific choice of maturation velocity, we introduce an intermediate notation $\bar{h}(u, y)$ so that $h(t, x, y)=\bar{h}(u(t), y)$ for $x \in G_{1} \cup D$, with

$$
\bar{h}(u, y)=\tau_{h}^{f}\left[-y^{2}+\left(c_{1}^{f} y+c_{2}^{f}\right)\left(1-e^{-\frac{u}{\bar{u}^{f}}}\right)\right],
$$

and we denote by $y_{1}(u) \leq 0 \leq y_{2}(u)$ the two roots of $\bar{h}(u, y)=0$. Relaxing hypothesis (3.6) made on $h[26]$ leads us to prove the following Lemma

Lemma C.1. Suppose that

$$
c_{1} \theta\left(u_{\max }\right)<c_{1} \theta\left(u_{\min }\right)+\sqrt{\left(c_{1} \theta\left(u_{\min }\right)\right)^{2}+4 c_{2} \theta\left(u_{\min }\right)}
$$

where

$$
\theta(u)=1-\exp (u / \bar{u})
$$

Then there exists $0<y_{0_{\max }}<y_{2}\left(u_{\min }\right)<\infty$ and $\eta>0$ such that

$$
\frac{\partial}{\partial y} \bar{h}(u, y) \leq-\eta, \quad \forall y \geq y_{0_{\max }}, \quad \forall u, u_{\min } \leq u \leq u_{\max } .
$$

Proof. From (C.1) we have that

$$
\frac{\partial}{\partial y} \bar{h}(u, y)=\tau_{h}\left(-2 y+c_{1} \theta(u(t))\right)
$$

therefore

$$
\frac{\partial}{\partial y} \bar{h}(u, y)=0 \Leftrightarrow y=y_{0}(u):=\frac{c_{1} \theta(u)}{2} .
$$

Furthermore, since $\theta(u)$ in increasing, we have that

$$
\begin{aligned}
\frac{\partial}{\partial y} \bar{h}(u, y) & \leq \frac{\partial}{\partial y} \bar{h}\left(u_{\max }, y\right) \quad \forall u \leq u_{\max } \\
& \leq \frac{\partial}{\partial y} \bar{h}\left(u_{\max }+\varepsilon, y_{0}\left(u_{\max }\right)\right) \quad \forall y \geq y_{0}\left(u_{\max }\right), \quad \forall u \leq u_{\max }+\varepsilon \\
& \leq \frac{\partial}{\partial y} \bar{h}\left(u_{\max }+\varepsilon, y_{0}\left(u_{\max }+\varepsilon\right)\right) \quad \forall y \geq y_{0}(u), \quad \forall u \leq u_{\max }+\varepsilon
\end{aligned}
$$

Therefore for all $y \geq y_{0}\left(u_{\max }+\varepsilon\right)$, and for all $u \leq u_{\max }$

$$
\frac{\partial}{\partial y} \bar{h}(u, y) \leq \frac{\partial}{\partial y} \bar{h}\left(u_{\max }, y_{0}\left(u_{\max }+\varepsilon\right)\right)=-\eta<0 .
$$

This means that for fixed $u \leq u_{\max }, \bar{h}(u, y)$ is decreasing for all $y \geq y_{0_{\max }}:=y_{0}\left(u_{\max }+\right.$ $\varepsilon)$ (see Figure C.1).

For any $u, u_{\min } \leq u \leq u_{\max }$, the function $\bar{h}(u, y)=\tau_{h}\left(-y^{2}+\left(c_{1} y+c_{2}\right) \theta(u)\right)$ has two distinct roots $y_{1}(u), y_{2}(u)$ such that

$$
y_{1}(u)<0<y_{0}(u)<y_{2}(u) .
$$


Precisely, we have that

$$
y_{2}(u)=\frac{c_{1} \theta(u)+\sqrt{\left(c_{1} \theta(u)\right)^{2}+4 c_{2} \theta(u)}}{2},
$$

with $\theta(u)$ defined in (C.3). Let us set $y_{2_{\min }}:=y_{2}\left(u_{\min }\right)$, and check whether we can choose $\varepsilon>0$ such that $y_{2_{\min }} \geq y_{0_{\max }}$. Since $\theta(u)$ is continuous and strictly increasing this is equivalent to check that $y_{2_{\min }}>y_{0}\left(u_{\max }\right)$ which boils down to (C.2). $\square$ There exist values for $c_{1}, c_{2}, \bar{u}$ such that condition (C.2) is satisfied and Figure C.1 displays characteristic maturities for an instance of $\bar{h}(u, y)$. We then enunciate properties of the 2D and 1D solutions which will used to obtain the long time behaviour.

Lemma C.2. Suppose that $\Phi$ is a solution of (2.10) satisfying (3.7) and that $\zeta(t, x)$ is a solution of (3.1) with

$$
\zeta(0, x)=\zeta^{0} \in\left[y_{\min }^{0}, y_{\max }^{0}\right] .
$$

Under hypotheses of Lemma C.1 there exist $0<t_{s}<\infty, y_{0}\left(t_{s}\right)$, and $y_{1}\left(t_{s}\right)$, such that

$$
\operatorname{Supp}\left(\Phi\left(t=t_{s}, . .\right)\right) \subset\left[0, x_{2}\right] \times\left[y_{0}\left(t_{s}\right), y_{1}\left(t_{s}\right)\right],
$$

with $y_{0_{\max }} \leq y_{0}\left(t_{s}\right)<y_{1}\left(t_{s}\right)<y_{\max }$ and

$$
\zeta(t, x)>y_{0_{\max }} \quad \forall t \geq t_{s} .
$$

Proof. Looking at Figure C.1, we see that $\bar{h}(u, y)) \geq 0$ for $y \leq y_{2_{\min }}=y_{0}^{s}$ and

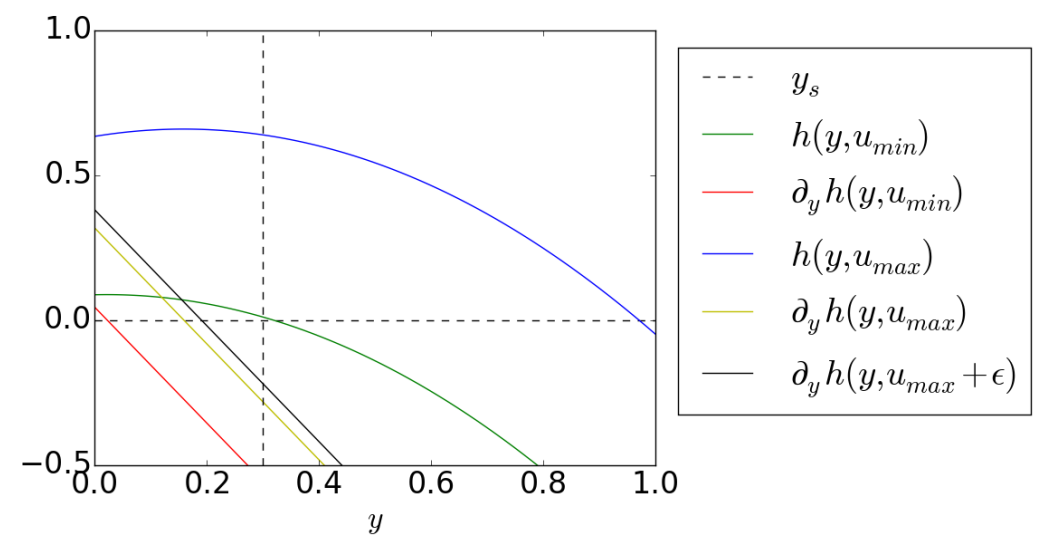

FIG. C.1. Functions $\bar{h}(u, y)$ for $u=b_{1} U_{\max }$ and $u=U_{\max }$, and partial derivative $\partial_{y} \bar{h}(u, y)$ for $u=b_{1} U_{\max }, U_{\max }$ and $U_{\max }+\epsilon$. For parameter values $c_{1}=0.6, c_{2}=1.2, \bar{u}=0.2$ and $b_{1}=0.2$, $\epsilon=0.05$ is a satisfying value. The $x$-coordinate of the intersection between the green curve and the black straight line is $y_{2 \text { min }}$. The $\mathrm{x}$-coordinate of the intersection between the yellow and black lines is $y_{0 \max }$.

$y_{2_{\min }}>y_{0_{\max }}$ (with $y_{0_{\max }}$ defined in Lemma C.1). Then the support of $\phi(t, x, y)$ is transported upward in the $y$ direction until at least $y_{2_{\min }}$, since the speed is positive for all $u$ up to this maturity. Since $y_{2_{\min }}>y_{0_{\max }}$, the speed is strictly positive for all $u$ until $y \geq y_{0_{\max }}$. Hence, starting from the initial support (3.7), this maturity is reached for $t_{y}<\infty$. From the monotonicity of $y_{2}(u)$ we also get a bound $y_{1}^{s} \leq y_{2}\left(u_{\max }\right) \leq y_{\max }$ for the upper limit of the domain $\Omega$. 
Similarly, considering the $\operatorname{PDE}(3.1)$ of $\zeta(t, x)$, since $\bar{h}(u, \zeta) \geq 0$ for $\zeta \leq y_{2_{\min }}$ and $y_{2_{\min }}>y_{0_{\max }}$, there exists $t_{\zeta}>0$ such that $\zeta(t, x)>y_{0_{\max }}$ for all $t \geq t_{\zeta}$.

Denoting $t_{s}=\max \left(t_{y}, t_{\zeta}\right)$, we have that

$$
\begin{aligned}
& h(t, x, y)=\bar{h}(u(t), y)>0 \text { for } 0<x<x_{1}, 0<y<y_{0}(u(t)), t \leq t_{s}, \\
& h(t, x, y)=0 \text { for } x_{1}<x<x_{2}, 0<y<y_{s} .
\end{aligned}
$$

Therefore

$$
\operatorname{Supp}\left(\Phi\left(t=t_{s}\right)\right) \subset\left[0, x_{2}\right] \times\left[y_{0}^{s}, y_{1}^{s}\right],
$$

where $y_{0_{\max }} \leq y_{0}^{s}<y_{1}^{s}<y_{\max }$. This completes the proof of Lemma C.2. In order to prove Theorem 3.3, we introduce the duality pair $\Phi, \psi$ where $\psi>0$ in $C^{1}\left(\left[0, T,\left[0, x_{2}\right],\left[0, y_{\max }\right]\right)\right.$ for $T>0$ is a solution of the dual (backward) equation

$$
\left\{\begin{array}{l}
\frac{\partial}{\partial t} \psi+g \frac{\partial}{\partial x} \psi+h \frac{\partial}{\partial y} \psi=\Lambda \psi \\
\psi(t, 0, y)=\frac{1}{2} \psi\left(t, x_{2}, y\right) \quad \text { if } y \leq y_{s}, \\
\psi(t, 0, y)=\psi\left(t, x_{2}, y\right) \quad \text { if } y>y_{s} \\
\psi(t, x, 0)=1+\frac{x}{x_{2}}, \\
\psi(T, x, y)=1+\frac{x}{x_{2}} .
\end{array}\right.
$$

In [26], where velocities $g$ and $h$ are continuous, the existence of $\psi$ is ensured by Theorem 6.1 in Chapter 6 of [29]. The discontinuous set-up is tackled in [30] by a rescaling of the different subdomains where the coefficients are continuous. We can also rely on a more general result for transport equations with locally positive vector field [12]. We prove in Appendix D that $\psi$ furthermore satisfies the properties stated in the following lemma

Lemma C.3. Let $F:(-\infty, \infty) \rightarrow[0, \infty)$ with $C^{1}$ regularity. Let $\zeta, \psi, \Phi$ be solutions to (3.1), (C.3), (2.10). Then we have the conservation law

$$
\left\{\begin{array}{l}
\iint_{\Omega} \Phi(t, x, y) \psi(t, x, y) d x d y=C s t \\
\frac{d}{d t} \iint_{\Omega} \Phi(t, x, y) \psi(t, x, y) F(y-\zeta(t, x)) d x d y= \\
\iint_{\Omega} \Phi(t, x, y) \psi(t, x, y) F^{\prime}(y-\zeta(t, x))[h(t, x, y)-h(t, x, \zeta(t, x))] d x d y
\end{array}\right.
$$

Proof. The proof of Theorem 3.3 starts by applying (C.9) with $F(x)=x^{2}$. We have

$$
\begin{gathered}
\frac{d}{d t} \iint_{\Omega} \Phi \psi(y-\zeta(t, x))^{2} \mathrm{~d} x \mathrm{~d} y=2 \iint_{\Omega} \Phi \psi(y-\zeta(t, x))[h(x, \zeta(t, x), u(t)) \\
-h(x, y, u(t))] \mathrm{d} x \mathrm{~d} y
\end{gathered}
$$

From Lemma C.2, when $t \geq t_{s}$, both $y$ and $\zeta(t, x)$ are in $\left[y_{0}^{s}, y_{1}^{s}\right]$. Then, applying Taylor Theorem and (C.4), there exist $y_{l}$ between $y$ and $\zeta(t, x)$ such that

$$
\begin{aligned}
h(x, \zeta(t, x), u(t))-h(x, y, u(t)) & =(y-\zeta(t, x)) \frac{\partial h}{\partial y}\left(x, y_{l}, u(t)\right), \\
& \leq-\eta(y-\zeta(t, x)) .
\end{aligned}
$$


Therefore, we get

$$
\frac{d}{d t} \iint_{\Omega} \Phi \psi(y-\zeta(t, x))^{2} \mathrm{~d} x \mathrm{~d} y \leq-2 \eta \iint_{\Omega} \Phi \psi(y-\zeta(t, x))^{2} \mathrm{~d} x \mathrm{~d} y \leq 0, \quad \forall t \geq t_{s} .
$$

Similarly, with $F(x)=\left(x_{+}\right)^{2}=\max (x, 0)^{2}$ and $F(x)=\left(x_{-}\right)^{2}=\min (x, 0)^{2}$, we have

$$
\begin{aligned}
& \frac{d}{d t} \iint_{\Omega} \Phi \psi\left((y-\zeta(t, x))_{+}\right)^{2} \mathrm{~d} x \mathrm{~d} y \leq-2 \eta \iint_{\Omega} \Phi \psi\left((y-\zeta(t, x))_{+}\right)^{2} \mathrm{~d} x \mathrm{~d} y \leq 0, \quad \forall t \geq t_{s} \\
& \frac{d}{d t} \iint_{\Omega} \Phi \psi\left((y-\zeta(t, x))_{-}\right)^{2} \mathrm{~d} x \mathrm{~d} y \leq-2 \eta \iint_{\Omega} \Phi \psi\left((y-\zeta(t, x))_{-}\right)^{2} \mathrm{~d} x \mathrm{~d} y \leq 0, \quad \forall t \geq t_{s}
\end{aligned}
$$

Thus, $\iint_{\Omega} \Phi \psi\left((y-\zeta(t, x))_{+}\right)^{2} \mathrm{~d} x \mathrm{~d} y$ and $\iint_{\Omega} \Phi \psi\left((y-\zeta(t, x))_{-}\right)^{2} \mathrm{~d} x \mathrm{~d} y$ are decreasing on $\left[t_{s}, \infty\right]$, therefore, for all $t \geq t_{s}$,

$$
\begin{gathered}
\iint_{\Omega} \Phi(t, x, y) \psi(t, x, y)\left((y-\zeta(t, x))_{-}\right)^{2} \mathrm{~d} x \mathrm{~d} y \leq \\
\iint_{\Omega} \Phi\left(t_{s}, x, y\right) \psi\left(t_{s}, x, y\right)\left(\left(y-\zeta\left(t_{s}, x\right)\right)_{-}\right)^{2} \mathrm{~d} x \mathrm{~d} y, \\
\iint_{\Omega} \Phi(t, x, y) \psi(t, x, y)\left((y-\zeta(t, x))_{+}\right)^{2} \mathrm{~d} x \mathrm{~d} y \leq \\
\iint_{\Omega} \Phi\left(t_{s}, x, y\right) \psi\left(t_{s}, x, y\right)\left(\left(y-\zeta\left(t_{s}, x\right)_{+}\right)^{2} \mathrm{~d} x \mathrm{~d} y .\right.
\end{gathered}
$$

We have, thanks to Lemma C.2

$$
\begin{gathered}
\iint_{\Omega} \Phi \psi\left(t_{s}, x, y\right)\left(\left(y-\zeta_{0}^{s}\left(t_{s}, x\right)\right)_{-}\right)^{2} \mathrm{~d} x \mathrm{~d} y=0, \\
\iint_{\Omega} \Phi \psi\left(t_{s}, x, y\right)\left(\left(y-\zeta_{1}^{s}\left(t_{s}, x\right)_{+}\right)^{2} \mathrm{~d} x \mathrm{~d} y=0 .\right.
\end{gathered}
$$

Hence, from (C.10,C.11) we get, for all $t \geq t_{s}$,

$$
\begin{aligned}
& \iint_{\Omega} \Phi \psi(t, x, y)\left(\left(y-\zeta_{0}^{s}(t, x)\right)_{-}\right)^{2} \mathrm{~d} x \mathrm{~d} y=0, \\
& \iint_{\Omega} \Phi \psi(t, x, y)\left(\left(y-\zeta_{1}^{s}(t, x)\right)_{+}\right)^{2} \mathrm{~d} x \mathrm{~d} y=0 .
\end{aligned}
$$

On the other hand, we have $\psi(t, x, y)>0$ for all $t>0$, age $x$ and maturity $y$ as soon as $\psi(0, x, y)>0$, therefore, for any $(x, y) \in \operatorname{Supp} \Phi, \quad \Phi \psi(t, x, y) \neq 0$. Furthermore, for $(x, y) \in \operatorname{Supp}(\Phi \psi)$, from (C.12) we get $\left(y-\zeta_{0}^{s}(t, x)\right)_{-}^{2}=0$ which is equivalent to $y \geq \zeta_{0}^{s}(t, x)$; and similarly from (C.13), we get $y \leq \zeta_{1}^{s}(t, x)$. Thus,

$$
\operatorname{Supp}(\Phi(t, . . .)) \subset\left\{(x, y), x \in\left[0, x_{2}\right], y \in\left[\zeta_{0}^{s}(t, x), \zeta_{1}^{s}(t, x)\right]\right\}, \quad \forall t \geq t_{s} .
$$


From here on, our proof is original compared to [26]. Rearranging terms and using the PDE (3.1), we have that

$$
\frac{\partial}{\partial t}\left(\zeta_{0}^{s}-\zeta_{1}^{s}\right)^{2}+g \frac{\partial}{\partial x}\left(\zeta_{0}^{s}-\zeta_{1}^{s}\right)^{2}=2\left(\zeta_{0}^{s}-\zeta_{1}^{s}\right)\left(h\left(x, \zeta_{0}^{s}, u\right)-h\left(x, \zeta_{1}^{s}, u\right)\right) .
$$

When $t \geq t_{s}$, both $\zeta_{0}^{s}$ and $\zeta_{1}^{s}$ are in $\left[y_{0_{\max }}, y_{\max }\right]$. Applying Taylor Theorem, we have that there exists $y_{l}$ between $\zeta_{0}^{s}$ and $\zeta_{1}^{s}$ such that

$$
\frac{\partial}{\partial t}\left(\zeta_{0}^{s}-\zeta_{1}^{s}\right)^{2}+g \frac{\partial}{\partial x}\left(\zeta_{0}^{s}-\zeta_{1}^{s}\right)^{2}=2\left(\zeta_{0}^{s}-\zeta_{1}^{s}\right)^{2} \frac{\partial h}{\partial y}\left(x, y_{l}, u\right) \leq-2 \eta\left(\zeta_{0}^{s}-\zeta_{1}^{s}\right)^{2} .
$$

Therefore, we get

$$
\int_{0}^{x_{2}}\left[\frac{\partial}{\partial t}\left(\zeta_{0}^{s}-\zeta_{1}^{s}\right)^{2}+g \frac{\partial}{\partial x}\left(\zeta_{0}^{s}-\zeta_{1}^{s}\right)^{2}\right] \mathrm{d} x \leq-\int_{0}^{x_{2}} 2 \eta\left(\zeta_{0}^{s}-\zeta_{1}^{s}\right)^{2} \mathrm{~d} x
$$

which is equivalent to

$$
\begin{aligned}
\frac{d}{d t} \int_{0}^{x_{2}}\left(\zeta_{0}^{s}-\zeta_{1}^{s}\right)^{2} \mathrm{~d} x+g\left[\left(\zeta_{0}^{s}-\zeta_{1}^{s}\right)^{2}(x\right. & \left.\left.=x_{2}\right)-\left(\zeta_{0}^{s}-\zeta_{1}^{s}\right)^{2}(x=0)\right] \\
& \leq-2 \eta \int_{0}^{x_{2}}\left(\zeta_{0}^{s}-\zeta_{1}^{s}\right)^{2} \mathrm{~d} x
\end{aligned}
$$

and, since $\left(\zeta_{0}^{s}-\zeta_{1}^{s}\right)^{2}\left(x=x_{2}\right)=\left(\zeta_{0}^{s}-\zeta_{1}^{s}\right)^{2}(x=0)$, we get

$$
\frac{d}{d t} \int_{0}^{x_{2}}\left(\zeta_{0}^{s}-\zeta_{1}^{s}\right)^{2} \mathrm{~d} x \leq-2 \eta \int_{0}^{x_{2}}\left(\zeta_{0}^{s}-\zeta_{1}^{s}\right)^{2} \mathrm{~d} x
$$

Using Gronwall Lemma between $t_{s}$ and $t$ we obtain

$$
\left\|\zeta_{0}^{s}(t, x)-\zeta_{1}^{s}(t, x)\right\|_{L^{2}\left(\left[0, x_{2}\right]\right)} \leq\left\|y_{0}^{s}-y_{1}^{s}\right\|_{L^{2}\left(\left[0, x_{2}\right]\right)} e^{-\eta\left(t-t_{s}\right)}
$$

Letting $t \rightarrow \infty$, we get

$$
\lim _{t \rightarrow \infty}\left\|\zeta_{0}^{s}(t, x)-\zeta_{1}^{s}(t, x)\right\|_{L^{2}\left(\left[0, x_{2}\right]\right)}=0,
$$

which completes our proof.

\section{Appendix D. Proof of Lemma C.3.}

Proof. We adapt the idea of the proof in [26] to the new set-up with $\Omega=G 1 \cup$ $S M \cup D 1 \cup D 2$. We have that

$$
\begin{aligned}
& \frac{\partial}{\partial t}(\Phi \psi)+\frac{\partial}{\partial x}(g \Phi \psi)+\frac{\partial}{\partial y}(h \Phi \psi) \\
= & \Phi \frac{\partial}{\partial t} \psi+\psi \frac{\partial}{\partial t} \Phi+g \Phi \frac{\partial}{\partial x} \psi+\psi \frac{\partial}{\partial x}(g \Phi)+h \Phi \frac{\partial}{\partial y} \psi+\psi \frac{\partial}{\partial y}(h \Phi) \\
= & \left.\Phi\left(\frac{\partial}{\partial t} \psi+g \frac{\partial}{\partial x} \psi+h \frac{\partial}{\partial y} \psi\right)+\psi\left(\frac{\partial}{\partial t} \Phi+\frac{\partial}{\partial x}(g \Phi)+\frac{\partial}{\partial y}(h \Phi)\right)\right) \\
= & \Phi(\Lambda \psi)+\psi(-\Lambda \Phi)=0
\end{aligned}
$$


Therefore

$$
\iint_{\Omega}\left(\frac{\partial}{\partial t}(\Phi \psi)+\frac{\partial}{\partial x}(g \Phi \psi)+\frac{\partial}{\partial y}(h \Phi \psi)\right) d x d y=0,
$$

which is equivalent to

$$
\begin{array}{r}
\frac{d}{d t} \iint_{\Omega} \Phi \psi d x d y+\int_{0}^{x_{1}} \int_{0}^{y_{s}} \frac{\partial}{\partial x}(g \Phi \psi) d x d y+\int_{x_{1}}^{x_{2}} \int_{0}^{y_{s}} \frac{\partial}{\partial x}(g \Phi \psi) d x d y \\
+\int_{0}^{x_{1}} \int_{y_{s}}^{y_{\max }} \frac{\partial}{\partial x}(g \Phi \psi) d x d y+\int_{x_{1}}^{x_{2}} \int_{y_{s}}^{y_{\max }} \frac{\partial}{\partial x}(g \Phi \psi) d x d y \\
\quad+\int_{0}^{x_{1}} \int_{0}^{y_{s}} \frac{\partial}{\partial y}(h \Phi \psi) d x d y+\int_{x_{1}}^{x_{2}} \int_{0}^{y_{s}} \frac{\partial}{\partial x}(h \Phi \psi) d x d y \\
+\int_{0}^{x_{1}} \int_{y_{s}}^{y_{\max }} \frac{\partial}{\partial y}(h \Phi \psi) d x d y+\int_{x_{1}}^{x_{2}} \int_{y_{s}}^{y_{\max }} \frac{\partial}{\partial x}(h \Phi \psi) d x d y=0
\end{array}
$$

This implies that

$$
\begin{array}{r}
\frac{d}{d t} \iint_{\Omega} \Phi \psi d x d y+\int_{0}^{y_{s}}\left[g \Phi\left(t, x_{1}^{-}, y\right) \psi\left(t, x_{1}^{-}, y\right)-g \Phi(t, 0, y) \psi(t, 0, y)\right] d y \\
\quad+\int_{0}^{y_{s}}\left[g \Phi\left(t, x_{2}, y\right) \psi\left(t, x_{2}, y\right)-g \Phi\left(t, x_{1}^{+}, y\right) \psi\left(t, x_{1}^{+}, y\right)\right] d y \\
\quad+\int_{y_{s}}^{y_{\max }}\left[g \Phi\left(t, x_{1}^{-}, y\right) \psi\left(t, x_{1}^{-}, y\right)-g \Phi(t, 0, y) \psi(t, 0, y)\right] d y \\
+\int_{y_{s}}^{y_{\max }}\left[g \Phi\left(t, x_{2}, y\right) \psi\left(t, x_{2}, y\right)-g \Phi\left(t, x_{1}^{+}, y\right) \psi\left(t, x_{1}^{+}, y\right)\right] d y \\
\quad+\int_{0}^{x_{1}}\left[h \Phi\left(t, x, y_{s}^{-}\right) \psi\left(t, x, y_{s}^{-}\right)-h \Phi(t, x, 0) \psi(t, x, 0)\right] d x \\
+\int_{0}^{x_{1}}\left[h \Phi\left(t, x, y_{\max }\right) \psi\left(t, x, y_{\max }\right)-h \Phi\left(t, x, y_{s}^{+}\right) \psi\left(t, x, y_{s}^{+}\right)\right] d x \\
+\int_{x_{1}}^{x_{2}}\left[h \Phi\left(t, x, y_{\max }\right) \psi\left(t, x, y_{\max }\right)-h \Phi\left(t, x, y_{s}^{+}\right) \psi\left(t, x, y_{s}^{+}\right)\right] d x=0
\end{array}
$$

which leads to

$$
\frac{d}{d t} \iint_{\Omega} \Phi \psi d x d y-\int_{x_{1}}^{x_{2}} h \Phi\left(t, x, y_{s}^{+}\right) \psi\left(t, x, y_{s}^{+}\right) d x=0 .
$$

Since $\Phi\left(t, x, y_{s}^{+}\right)=0, \forall x \in\left[x_{1}, x_{2}\right]$, we get

$$
\frac{d}{d t} \iint_{\Omega} \Phi \psi d x d y=0
$$

which is equivalent to

$$
\iint_{\Omega} \Phi \psi d x d y=C s t
$$


For $F:(-\infty, \infty) \rightarrow[0, \infty)$ with $C^{1}$ regularity, we have

$$
\begin{aligned}
& \frac{\partial}{\partial t}(\Phi \psi F(y-\zeta(t, x)))+\frac{\partial}{\partial x}(g \Phi \psi F(y-\zeta(t, x)))+\frac{\partial}{\partial y}(h \Phi \psi F(y-\zeta(t, x))) \\
= & F(y-\zeta(t, x)) \frac{\partial}{\partial t}(\Phi \psi)-\Phi \psi \frac{\partial}{\partial t} F^{\prime}(y-\zeta(t, x)) \\
& +F(y-\zeta(t, x)) \frac{\partial}{\partial x}(g \Phi \psi)-g \Phi \psi \frac{\partial}{\partial x} \zeta(t, x) F^{\prime}(y-\zeta(t, x)) \\
& +F(y-\zeta(t, x)) \frac{\partial}{\partial y}(h \Phi \psi)+h \Phi \psi F^{\prime}(y-\zeta(t, x)), \\
= & F(y-\zeta(t, x))\left[\frac{\partial}{\partial t}(\Phi \psi)+\frac{\partial}{\partial x}(g \Phi \psi)+\frac{\partial}{\partial y}(h \Phi \psi)\right] \\
& -\Phi \psi F^{\prime}(y-\zeta(t, x))\left[\frac{\partial}{\partial t} \zeta(t, x)+g \frac{\partial}{\partial x} \zeta(t, x)-h(t, x, y)\right], \\
= & -\Phi \psi F^{\prime}(y-\zeta(t, x))[h(t, x, \zeta(t, x))-h(t, x, y)] .
\end{aligned}
$$

Therefore, we have

$$
\begin{aligned}
& \iint_{\Omega}\left(\frac{\partial}{\partial t}(\Phi \psi F(y-\zeta(t, x)))+\frac{\partial}{\partial x}(g \Phi \psi F(y-\zeta(t, x)))\right. \\
& \left.+\frac{\partial}{\partial y}(h \Phi \psi F(y-\zeta(t, x)))\right) d x d y \\
& =\iint_{\Omega} \Phi \psi F^{\prime}(y-\zeta(t, x))[h(t, x, \zeta(t, x))-h(t, x, y)] d x d y .
\end{aligned}
$$

Moreover, we have

$$
\begin{aligned}
& \iint_{\Omega} \frac{\partial}{\partial x}(g \Phi \psi F(y-\zeta(t, x)))+\frac{\partial}{\partial y}(h \Phi \psi F(y-\zeta(t, x))) d x d y \\
= & \int_{0}^{y_{s}}\left[g \Phi \psi\left(t, x_{1}^{-}, y\right) F\left(y-\zeta\left(t, x_{1}^{-}\right)\right)-g \Phi \psi(t, 0, y) F(y-\zeta(t, 0))\right] d y \\
+ & \int_{0}^{y_{s}}\left[g \Phi \psi\left(t, x_{2}, y\right) F\left(y-\zeta\left(t, x_{2}\right)\right)-g \Phi \psi\left(t, x_{1}^{+}, y\right) F\left(y-\zeta\left(t, x_{1}^{+}\right)\right)\right] d y \\
+ & \int_{y_{s}}^{y_{\max }}\left[g \Phi \psi\left(t, x_{1}^{-}, y\right) F\left(y-\zeta\left(t, x_{1}^{-}\right)\right)-g \Phi \psi(t, 0, y) F(y-\zeta(t, 0))\right] d y \\
+ & \int_{y_{s}}^{y_{\max }}\left[g \Phi \psi\left(t, x_{2}, y\right) F\left(y-\zeta\left(t, x_{2}\right)\right)-g \Phi \psi\left(t, x_{1}^{+}, y\right) F\left(y-\zeta\left(t, x_{1}^{+}\right)\right)\right] d y \\
+ & \int_{0}^{x_{1}}\left[h \Phi \psi\left(t, x, y_{s}^{-}\right) F\left(y_{s}^{-}-\zeta(t, x)\right)-h \Phi \psi(t, x, 0) F(-\zeta(t, x))\right] d x \\
+ & \int_{x_{1}}^{x_{2}}\left[h \Phi \psi\left(t, x, y_{s}^{-}\right) F\left(y_{s}^{-}-\zeta(t, x)\right)-h \Phi \psi(t, x, 0) F(-\zeta(t, x))\right] d x \\
+ & \int_{0}^{x_{1}}\left[h \Phi \psi\left(t, x, y_{\max }\right) F\left(y_{\max }-\zeta(t, x)\right)-h \Phi \psi\left(t, x, y_{s}^{+}\right) F\left(y_{s}^{+}-\zeta(t, x)\right)\right] d x \\
+ & \int_{x_{1}}^{x_{2}}\left[h \Phi \psi\left(t, x, y_{\max }\right) F\left(y_{\max }-\zeta(t, x)\right)-h \Phi \psi\left(t, x, y_{s}^{+}\right) F\left(y_{s}^{+}-\zeta(t, x)\right)\right] d x \\
= & 0
\end{aligned}
$$

Therefore, we get

$$
\begin{array}{r}
\frac{d}{d t} \iint_{\Omega} \Phi \psi F(y-\zeta(t, x)) d x d y= \\
\iint_{\Omega} \Phi \psi F^{\prime}(y-\zeta(t, x))[h(t, x, \zeta(t, x))-h(t, x, y)] d x d y,
\end{array}
$$


which completes the proof of Lemma.

Appendix E. Alternative conservative formulation. In the numerical simulations we have selected the natural choice of unknowns suggested by the ansatz (2.22). Nevertheless the 1D reduced model can also be expressed in terms of unknowns $\widetilde{\rho}$ and $\mu$ with $\mu=\widetilde{\rho} \zeta$, which satisfies the following $\mathrm{PDE}$

$$
\begin{aligned}
\frac{\partial}{\partial t} \mu(t, x)+\frac{\partial}{\partial x}(g(t, x) \mu(t, x)) & =\widetilde{\rho}(t, x) h\left(t, x, \frac{\mu(t, x)}{\widetilde{\rho}(t, x)}\right)-\Lambda\left(t, x, \frac{\mu(t, x)}{\widetilde{\rho}(t, x)}\right) \mu(t, x), \\
\mu\left(t, x_{1}^{+}\right) & =g\left(t, x_{1}^{-}\right) \mu\left(t, x_{1}^{-}\right) \\
\mu\left(t, 0^{+}\right) & = \begin{cases}2 \mu\left(t, x_{2}^{-}\right) & \text {if } \mu\left(t, x_{2}^{-}\right) \leq y_{s} \widetilde{\rho}\left(t, x_{2}^{-}\right) \\
\mu\left(t, x_{2}^{-}\right) & \text {otherwise }\end{cases}
\end{aligned}
$$

The conservative formulation (E.1), along with its counterpart (3.3) for $\widetilde{\rho}$, can be used to design a finite volume scheme with unknowns $\mu_{k}^{n}, \rho_{k}^{n}$. The discrete values of the solution at $t=0$ are initialized using a midpoint formula, accurate at the order 2 in space

$$
\mu_{k}^{0}=\zeta_{0}\left(x_{k+\frac{1}{2}}\right) \rho_{0}\left(x_{k+\frac{1}{2}}\right), \quad \rho_{k}^{0}=\rho_{0}\left(x_{k+\frac{1}{2}}\right) .
$$

Evolution scheme. Integration over $\left[t^{n}, t^{n+1}\right] \times\left[x_{k}, x_{k+1}\right]$ yields the first order numerical scheme. We obtain the following recursion on the approximate maturity and mass

$$
\begin{aligned}
\mu_{k}^{n+1}= & \mu_{k}^{n}-\frac{\Delta_{t}}{\Delta_{x}}\left(g\left(t^{n}, x_{k+1 / 2}\right) \mu_{k}^{n}-g\left(t^{n}, x_{k-1 / 2}\right) \mu_{k-1}^{n}\right)+\Delta_{t} h\left(t^{n}, x_{k},\right) \rho_{k}^{n} \\
& -\Delta_{t} \Lambda\left(t^{n}, x_{k+1 / 2}, \zeta_{k}^{n}\right) \mu_{k}^{n}, \\
\rho_{k}^{n+1}= & \rho_{k}^{n}-\frac{\Delta_{t}}{\Delta_{x}}\left[g\left(t^{n}, x_{k+1 / 2}\right) \rho_{k}^{n}-g\left(t^{n}, x_{k-1 / 2}\right) \rho_{k-1}^{n}\right] \\
& -\Delta_{t} \Lambda\left(t^{n}, x_{k+1 / 2}, \zeta_{k}^{n}\right) \rho_{k}^{n} \\
\zeta_{k}^{n+1}=\frac{\mu_{k}^{n+1}}{\rho_{k}^{n+1}} &
\end{aligned}
$$

The periodic or doubling conditions in (E.1) and (3.4) are discretized on the unknowns on meshes 0 and $N_{x}-1$ to compute $\zeta_{0}^{n+1}$ and $\rho_{0}^{n+1}$

$$
\begin{aligned}
\mu_{0}^{n+1}= & \mu_{0}^{n}-\frac{\Delta_{t}}{\Delta_{x}}\left(g\left(t^{n}, x_{1 / 2}\right) \mu_{0}^{n}-D g\left(t^{n}, x_{N_{x}-1 / 2}\right) \mu_{N_{x}-1}^{n}\right)+\Delta_{t} h\left(t^{n}, x_{1 / 2}, \zeta_{0}^{n}\right) \rho_{0}^{n} \\
& -\Delta_{t} \Lambda\left(t^{n}, x_{1 / 2}, \zeta_{0}^{n}\right) \mu_{0}^{n}, \\
\rho_{0}^{n+1}= & \rho_{0}^{n}-\frac{\Delta_{t}}{\Delta_{x}}\left[g\left(t^{n}, x_{1 / 2}\right) \rho_{0}^{n}-D g\left(t^{n}, x_{N_{x}-1 / 2}\right) \rho_{N_{x}-1}^{n}\right]-\Delta_{t} \Lambda\left(t^{n}, x_{1 / 2}, \zeta_{0}^{n}\right) \rho_{0}^{n} \\
\zeta_{0}^{n+1}= & \frac{\mu_{0}^{n+1}}{\rho_{0}^{n+1}} .
\end{aligned}
$$

with $D=2$ when $\zeta_{0}^{n}<y_{s}$, in order to satisfy the doubling (mitosis) condition, and $D=1$ when $\zeta_{0}^{n} \geq y_{s}$.

Figure E.1 displays the numerical accuracy of this scheme when used to compute the exact solution. The conclusions are similar to the results obtained for the non conservative scheme displayed in Figure 4.2. 

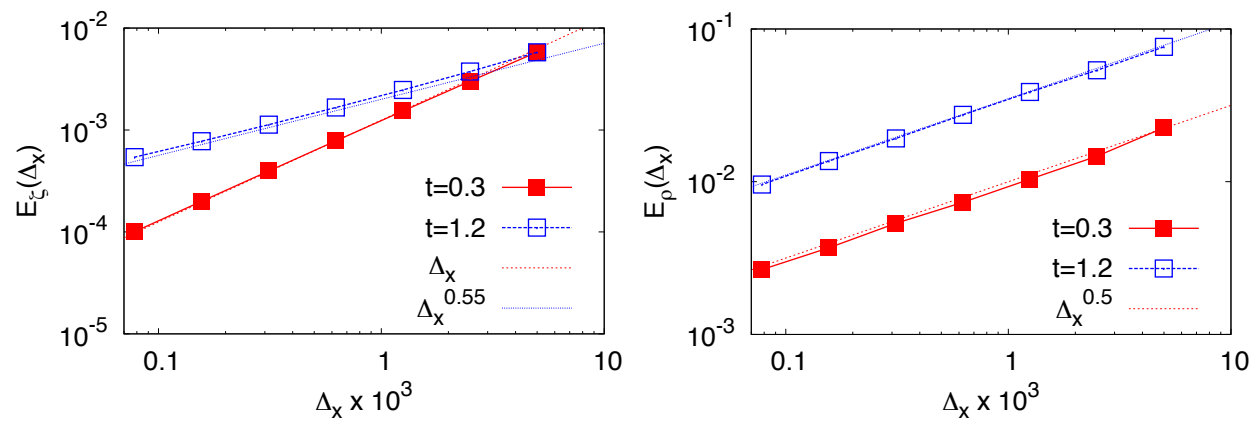

FIG. E.1. Relative error in $L_{1}$-norm between the exact and numerical $\zeta$ (left) and $\widetilde{\rho}$ (right) solutions at $t=0.3$ and $t=1.2$ with conservative cheme. 